NATIONAL AERONAUTICS AND SPACE ADMINISTRATION

Technical Report 32-1308

\title{
Elastic Wave Propagation for Plane Strain Problems by the Theory of Characteristics
}

M. Ziv

GPO PRICE

s QSETI PRICE(S) \$

Hard copy $(\mathrm{HC})$

Microfiche (MF) -
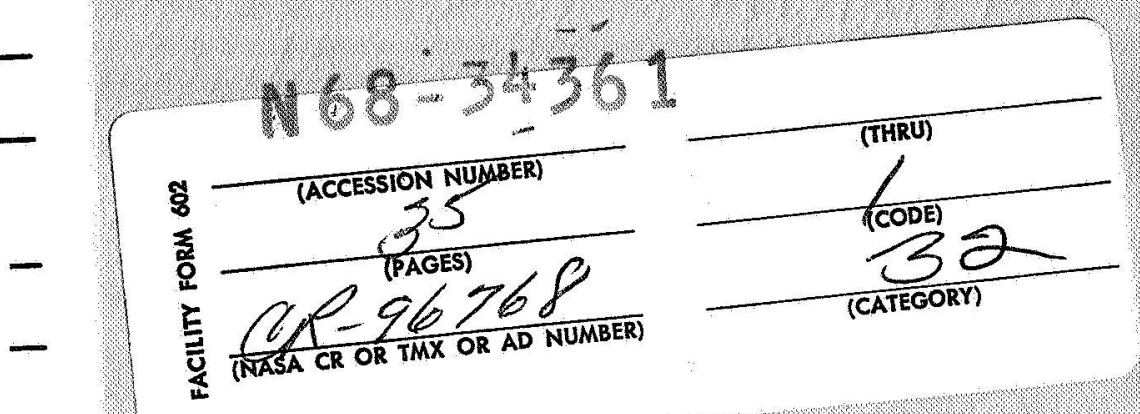

J E T P R O PULSION LA B O R A TOR Y CALIFORNIA INSTITUTE OF TECHNOLOGY PASA DENA, CALIFOR NIA

February 29,1968

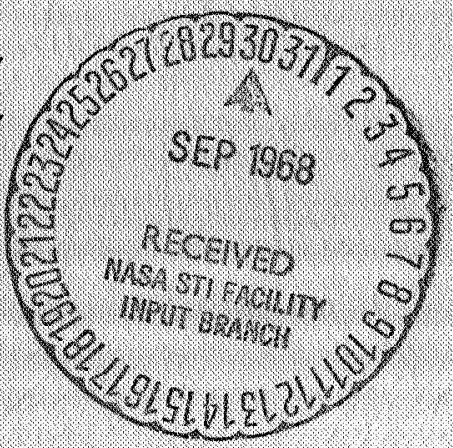




\section{Technical Report 32-1308}

\section{Elastic Wave Propagation for Plane Strain Problems by the Theory of Characteristics}

M. Ziv

Approved by:

m. E. Alpen Applied Mechanics Section

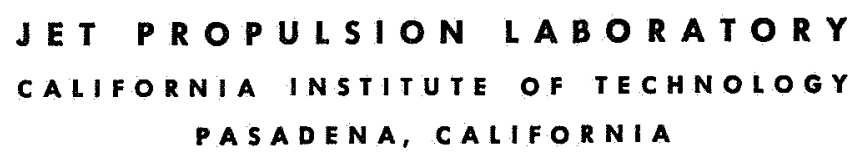

February 29, 1968 
TECHNICAL REPORT 32-1308

\author{
Copyright (C) 1968 \\ Jef Propulsion Laboratory \\ California Institute of Technology \\ Prepared Under Contract No. NAS 7-100
}

National Aeronautics \& Space Administration 


\section{Acknowledgment}

The author wishes to thank Mr. T. Lang and Dr. S. Utku for suggesting the topic, and Dr. Utku in particular for valuable discussions which helped provide insight into the field of wave propagation. Acknowledgment is also made to Mr. B. Wada for reading the report and for his revision suggestions. 


\section{Elastic Wave Propagation for Plane Strain Problems by the Theory of Characteristics}

\section{Introduction}

The phenomenon of transient wave propagation in solids has created increased interest in recent years. Solutions have been sought to wave problems, particularly those which arise in fields of geophysics and space technology. Some of the geophysical problems of current interest include protection from nuclear explosions and the detection of underground structures and minerals. In space technology, scientists encounter complex wave problems associated with meteorite impact on space vehicles, the impulsive ignition of solid-propellant motors, and impact landings of space vehicles. These problems generally require the treatment of finite bodies subjected to concentrated impulsive loads on one or more of their boundaries. Waves generated under these conditions are inherently multidimensional.

For a wide range of practical cases, multidimensional systems can be narrowed down to two-spatial dimensional waves. However, the solutions that can be obtained with existing techniques, even with this idealization, are quite limited. In the past, Fourier and Laplace transform techniques were the only methods applied to the solu- tion of two-spatial dimensional wave propagation problems in solids. Only few boundary-value problems have been solved using these techniques, and then only in the case of linear materials (Ref. 1). For example, within the last 60 years, researchers have treated the problem of a line load suddenly applied to a half-space using transform techniques, but found it exceedingly difficult to perform the numerical evaluation. The primary obstacle to these solutions has been the difficulty encountered in obtaining the inverse transform. As a result, use of the transform techniques has been limited to simple boundary-value problems. Furthermore, it was shown by Chou and Gordon that the Laplace transform technique fails in certain regions in the solution domain (Ref. 2).

Limitations and difficulties in the transform techniques encouraged researchers to apply the theory of characteristics to wave propagation problems. The theory of characteristics involves the solution of the basic dynamical field equations which govern the deformation in the medium. These field equations are first-order partialdifferential equations of the hyperbolic type. Their solutions are obtained by a conventional method used in the 
theory (Ref. 3). This method determines the differential equations which relate the dependent variables on the wave fronts, where discontinuities are assumed to occur in the derivatives of the variables. Once these differential equations are found, they are directly integrated numerically by the finite difference method. Because of the direct numerical integration, the theory of characteristics offers an advantage over the transform techniques, which require the inversion transform before integration can take place.

The theory of characteristics has proved relatively simple in its application to the one-spatial dimensional problem and has brought very successful results, particularly for the transient state (Ref. 4). Solutions have been obtained for materials that are elastic as well as nonelastic, homogeneous as well as nonhomogeneous, and linear as well as nonlinear (Refs. 5 and 6).

Since the theory of characteristics has been successfully applied to the solution of one-spatial dimensional wave problems in solids, it seems desirable to extend the theory to include two-spatial dimensional wave problems in solids. The failure to apply the theory of characteristics to solve such problems may be due to the inability to employ directly the conventional method, in which each distinct derivative in the basic dynamical field equations is considered to be an unknown. For numerous onespatial dimensional cases, the unknown derivatives are equal in number to the basic dynamical field equations available, thus making the conventional method applicable. This is not the case, however, in the two-spatial dimensional wave problems, where the number of the unknown derivatives exceeds the number of available basic dynamical equations. Obviously, the conventional method cannot be applied in this case. The motivation for this study is the need for a new method by which this type of two-spatial dimensional wave problem can be solved using the theory of characteristics.

In his work on surfaces of discontinuity, Hadamard developed a theory which presents these surfaces as the loci of discontinuities in the dependent variables and their derivatives (Ref. 7). In this theory, a surface divides a region of space into two parts. A function of space and time is considered to be continuous and differentiable on each side of the dividing surface, where the function and its derivatives may be discontinuous. Hence, this dividing surface is the discontinuity surface. Once the discontinuity surface affects different particles as it moves in a medium, it becomes a wave surface (also known as the wave front). In view of this model, kinematical conditions were derived by Hadamard for the function and its derivatives in reference to space and time, respectively, which are denoted as Hadamard's discontinuity relations. Since Hadamard's discontinuity relations were derived on a kinematical basis only, they seem to be applicable to a wide variety of constitutive equations, and the above function may then be replaced by velocities and stresses. This work was extended by Levi-Civita (Ref. 8) and T. Y. Thomas (Ref. 9). In particular, Thomas has done very valuable and extensive work on the subject within the last decade. Therefore, it seems expedient to employ Hadamard's work on surfaces of discontinuity in the effort to develop the new method by which twospatial dimensional wave problems in solids can be solved using the theory of characteristics.

The sections which follow describe these efforts. Hadamard's theory is applied to a wave surface across which the first partial derivatives of the velocities and stresses are discontinuous, while the dependent variables themselves are continuous. The discontinuity relations are reformulated in order to reduce the basic dynamical field equations to a system of dynamical conditions. The objective of this analysis is to present the dynamical conditions as a set of equations equal in number to the indeterminate (discontinuous) first partial derivatives. From the dynamical conditions, the differential equations governing the propagation of discontinuities on the wave surfaces are derived. These differential equations are referred to as the characteristic equations.

The characteristic equations are derived with reference first to Cartesian and then to cylindrical coordinate systems, thus allowing their application to a wide range of problems. As a measure of validity, the derived twospatial dimensional characteristic equations must be shown upon reduction to contain the well known onespatial dimensional form.

Finally, the derived characteristic equations are applied to the numerical evaluation of two representative boundary value problems. In both cases, an abrupt load is applied; therefore, special derivation is required in order to account for possible discontinuities in velocities and stresses across the leading wave front. The first problem considered consists of a uniform plane oblique load suddenly applied on an infinite flat plate. This problem, while one-spatial dimensional, involves two kinds of waves, namely, a longitudinal and a shear wave. Since the longitudinal and shear waves cause the material particles to move in two different directions, this system may 
be considered a step toward a strictly two-spatial dimensional problem. The second problem is two-spatial dimensional and consists of a line load suddenly applied on a half-space.

The investigation described in this report is confined to two-spatial dimensional wave propagation in a linear elastic, isotropic, and homogeneous material.

\section{Basic Dynamical Field Equations and the Monge Cone}

The basic dynamical field equations which govern the deformation in the medium are written below with respect to the Cartesian coordinate system. Total differentials of the dependent variables are then combined with the basic dynamical field equations to produce a characteristic surface.

The equations of motion for linear elastic material where plane strain is prevalent are

$$
\begin{aligned}
& \frac{\partial \boldsymbol{\sigma}_{x z}}{\partial x}+\frac{\partial \boldsymbol{\sigma}_{x z}}{\partial z}=\rho \frac{\partial^{2} \boldsymbol{u}}{\partial t^{2}} \\
& \frac{\partial \boldsymbol{\sigma}_{x z}}{\partial x}+\frac{\partial \boldsymbol{\sigma}_{z z}}{\partial z}=\rho \frac{\partial^{2} w}{\partial t^{2}}
\end{aligned}
$$

where

$$
\rho=\text { density }
$$

$u$ and $w=$ displacements in the $x$ - and $z$-directions, respectively

$\sigma_{x x}, \sigma_{z z}$, and $\sigma_{x z}=$ the normal and shear stresses in the $x, z$-plane

$x$ and $z=$ Cartesian coordinates

$$
t=\text { the time dimension }
$$

The constitutive equations for linear elastic, isotropic, and homogeneous material in terms of stress-displacement relations are

$$
\begin{aligned}
& \boldsymbol{\sigma}_{x x}=\lambda\left(\frac{\partial u}{\partial x}+\frac{\partial w}{\partial z}\right)+2 \mu \frac{\partial u}{\partial x} \\
& \boldsymbol{\sigma}_{z z}=\lambda\left(\frac{\partial u}{\partial x}+\frac{\partial w}{\partial z}\right)+2 \mu \frac{\partial w}{\partial z} \\
& \sigma_{x z}=\mu\left(\frac{\partial w}{\partial x}+\frac{\partial u}{\partial z}\right)
\end{aligned}
$$

where $\mu$ and $\lambda=$ Lamés constants.
In the theory of characteristics, it is convenient to treat first-order partial-differential equations, since they present directly algebraic relations among the first partial derivatives involved. Also, in many boundary-value problems which involve wave propagation, the prescribed conditions are in terms of forces or velocities or both. For the above reasons, Equations (3), (4); and (5) are differentiated with respect to time, and the terms $\partial u / \partial t$ and $\partial w / \partial t$ are replaced by $U$ and $W$, respectively. The $U$ and $W$ are velocities in the $x$ - and $z$-directions, respectively. The basic dynamical field equations now become a set of five linear first-order partial-differential equations with five dependent variables, $\sigma_{x x}, \sigma_{z z}, \sigma_{x z}, U$, and $W$, and with three independent variables, $x, z$, and $t$, as follows:

$$
\begin{gathered}
\frac{\partial \boldsymbol{\sigma}_{x x}}{\partial x}+\frac{\partial \boldsymbol{\sigma}_{z x}}{\partial z}=\rho \frac{\partial U}{\partial t} \\
\frac{\partial \boldsymbol{\sigma}_{x z}}{\partial x}+\frac{\partial \boldsymbol{\sigma}_{z z}}{\partial z}=\rho \frac{\partial W}{\partial t} \\
\frac{\partial \boldsymbol{\sigma}_{z x}}{\partial t}=\mu\left(\frac{\partial W}{\partial x}+\frac{\partial U}{\partial z}\right) \\
\frac{\partial \boldsymbol{\sigma}_{z z}}{\partial t}=\lambda\left(\frac{\partial U}{\partial x}+\frac{\partial W}{\partial z}\right)+2 \mu \frac{\partial W}{\partial z} \\
\frac{\partial \boldsymbol{\sigma}_{x x}}{\partial t}=\lambda\left(\frac{\partial U}{\partial x}+\frac{\partial W}{\partial z}\right)+2 \mu \frac{\partial U}{\partial x}
\end{gathered}
$$

In addition to the basic dynamical field equations (Eqs. 6 through 10), total differentials for the dependent variables are necessary to produce an integral surface (a solution surface), as evident from the following: Let a function $f$ stand for any of the dependent variables $\sigma_{x x}$, $\sigma_{z z}, \sigma_{x z}, U$, and $W$, and let $f$ and its first partial derivatives $\partial f / \partial x, \partial f / \partial z$, and $\partial f / \partial t$ be continuous in certain regions in the medium which occupies the space $x, z, t$. It is known that at each point in the medium, where the basic dynamical field equations are defined, there exists a Monge cone (Ref. 10). The Monge cone is formed by varying the first partial derivatives $\partial f / \partial x, \partial f / \partial z$, and $\partial f / \partial t$, which are algebraically related by the basic dynamical field equations. For a surface $f=f(x, z, t)$ to be an integral surface of the basic dynamical field equations, its tangent element should touch the Monge cone at each point in the field defined by the equations. The point $(x+d x, z+d z, t+d t, f+d f)$ lies in a tangent element to the Monge cone at the point $x, z, t, f$ if

$$
d f=(\partial f / \partial x) d x+(\partial f / \partial z) d z+(\partial f / \partial t) d t
$$


(Ref. 10). Consequently, the following total differentials must be introduced as

$$
\begin{aligned}
& d f=(\partial f / \partial x) d x+(\partial f / \partial z) d z+(\partial f / \partial t) d t \\
& d U=\frac{\partial U}{\partial x} d x+\frac{\partial U}{\partial z} d z+\frac{\partial U}{\partial t} d t \\
& d W=\frac{\partial W}{\partial x} d x+\frac{\partial W}{\partial z} d z+\frac{\partial W}{\partial t} d t \\
& d \sigma_{x z}=\frac{\partial \sigma_{x z}}{\partial x} d x+\frac{\partial \sigma_{x z}}{\partial z} d z+\frac{\partial \sigma_{x z}}{\partial t} d t \\
& d_{\sigma_{z z}}=\frac{\partial \sigma_{z z}}{\partial x} d x+\frac{\partial \sigma_{z z}}{\partial z} d z+\frac{\partial \sigma_{z z}}{\partial t} d t \\
& d_{\sigma_{x x}}=\frac{\partial \sigma_{x x}}{\partial x} d x+\frac{\partial \sigma_{x x}}{\partial z} d z+\frac{\partial \sigma_{x x}}{\partial t} d t
\end{aligned}
$$

Equations (6) through (15) are combined to eliminate first partial derivatives with respect to time and are now presented as follows:

$$
\begin{aligned}
& \rho \frac{\partial U}{\partial x} \frac{d x}{d t}+\rho \frac{\partial U}{\partial z} \frac{d z}{d t}+\frac{\partial \sigma_{x x}}{\partial x}+\frac{\partial \sigma_{z x}}{\partial z}=\rho \frac{d U}{d t} \\
& \rho \frac{\partial W}{\partial x} \frac{d x}{d t}+\rho \frac{\partial W}{\partial z} \frac{d z}{d t}+\frac{\partial \sigma_{x z}}{\partial x}+\frac{\partial \sigma_{z z}}{\partial z}=\rho \frac{d W}{d t}
\end{aligned}
$$

$$
\frac{\partial \sigma_{z x}}{\partial x} \frac{d x}{d t}+\frac{\partial \sigma_{z x}}{\partial z} \frac{d z}{d t}+\mu \frac{\partial W}{\partial x}+\mu \frac{\partial U}{\partial z}=\frac{d \sigma_{z x}}{d t}
$$

$$
\frac{\partial \boldsymbol{\sigma}_{z z}}{\partial x} \frac{d x}{\boldsymbol{d} t}+\frac{\partial \boldsymbol{\sigma}_{z z}}{\partial z} \frac{d z}{d t}+\lambda \frac{\partial U}{\partial x}+\lambda \frac{\partial W}{\partial z}+2 \mu \frac{\partial W}{\partial z}=\frac{d \sigma_{z z}}{d t}
$$

$$
\frac{\partial \sigma_{x x}}{\partial x} \frac{d x}{d t}+\frac{\partial \sigma_{x x}}{\partial z} \frac{d z}{d t}+\lambda \frac{\partial U}{\partial x}+\lambda \frac{\partial W}{\partial z}+2 \mu \frac{\partial U}{\partial x}=\frac{d \sigma_{x x}}{d t}
$$

This system of differential equations will be referred to as the dynamical field equations.

The dynamical field equations (Eqs. 16 through 20) are shown in Section $\mathrm{V}$ to possess five real and distinct integral surfaces. Consequently, the dynamical field equa- tions are of the hyperbolic type and their integral surfaces are known (Ref. 10) as characteristic surfaces.

A characteristic surface starts to form when a point $P_{0}\left(x_{0}, z_{0}, t_{0}\right)$ in the medium in which the dynamical field equations are defined is excited by one or more of the dependent variables. To show the formation of an arbitrary characteristic surface by its tangent elements for an arbitrary point $P_{0}\left(x_{0}, z_{0}, t_{0}\right)$, the Monge cone with $P_{0}$ as its apex is considered (Fig. 1). Since each of the tangent elements of the characteristic surface must touch the Monge cone at point $P_{0}$, these elements will form a pyramid around the cone. The envelope of this pyramid is the characteristic surface. The generators of the envelope coincide with the pyramid along the curves formed by the intersection of the tangent elements. These curves are known as the bicharacteristic curves. Since the bicharacteristic curves are formed by tangent elements with different orientations, discontinuities may occur in the first partial derivatives across the bicharacteristic curves. Therefore, the first partial derivatives on the characteristic surface are indeterminate. Accordingly, the characteristic surfaces are surfaces of discontinuity in the first partial derivatives of the dependent variables. When point $P_{0}$ is excited by given initial conditions which are functions of time, the surface of discontinuity is propagated in the space $x, z, t$ as shown in Fig. 1 , and so are the indeterminate first partial derivatives along the bicharacteristic curves. The solution surface is then found by the propagation of these indeterminate first partial derivatives from the initial conditions along the bicharacteristic curves.

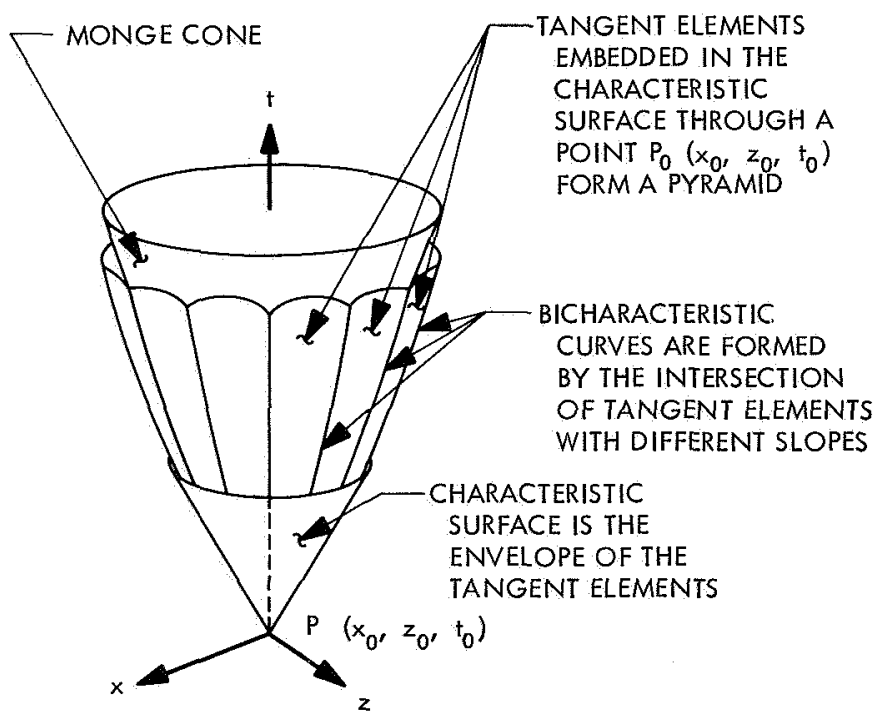

Fig. 1. Characteristic surfaces and bicharacteristic curves 
The objectives now are first to depict the surface of discontinuity as a wave surface and then to establish the dynamical conditions for the indeterminate first partial derivatives. The dynamical conditions are to be established from the dynamical field equations ( 16 through 20 ). From the dynamical conditions, a characteristic condition is to be found, which should yield the discontinuity surfaces and their bicharacteristic curves, along which the indeterminate first partial derivatives are propagating. Next, the characteristic equations which relate the dependent variables along the bicharacteristic curves are to be derived. Once these objectives have been reached, the propagation of the solution surface can be found when a point is excited by given initial conditions. The following sections are devoted to these objectives.

\section{Waves as Surfaces of Discontinuity}

Analytical tools are now being sought to treat waves as surfaces of discontinuity. The discussion at the end of Section II described the geometrical meaning and formation of surfaces of discontinuity from the dynamical field equations (16 through 20). Based on this notion, a model is defined here in which waves are depicted as surfaces of discontinuity. Once this model is defined, the theory on surfaces of discontinuity based on Hadamard (Refs. 7, 8, and 9) is introduced to present the discontinuity relations which hold across the wave surface.

The model considered is a surface $\Phi(x, z, t)=0$ in the medium. Two basic conditions are imposed on this surface: (1) It is required that the surface present the locus of possible discontinuities in the first partial derivatives of the dependent variables appearing in the dynamical field equations. The dependent variables themselves are assumed to be continuous everywhere, but differentiable only in regions which are in the rear and in the front of the discontinuity surface. (2) It is required that this surface of discontinuity present a wave surface in the sense that it affects different material particles as it moves in the medium.

From these basic conditions and from the dynamical field equations (16 through 20), it follows that the quantities $\partial U / \partial x, \partial U / \partial z, \partial W / \partial x, \partial W / \partial z$, and $\sigma_{i j, i}$ across the wave surface $\Phi$ are assumed to be discontinuous, but $p, \sigma_{i j}, U$, and $W$ are assumed to be continuous.*

\footnotetext{
"Whenever it is convenient, Cartesian tensor notations are used in this report; $\sigma_{i j}$ stands for the stress components $\sigma_{x x}, \sigma_{z z}$, and $\sigma_{x z}$. The comma in the term $\sigma_{i j, i}$ means partial differentiation with respect to $x$ and $z$.
}

Before formulation of the above model is attempted, a digression is made to show that the conventional method used in the theory of characteristics (Ref. 3) cannot be applied here. With the conventional method, the characteristic equations are derived directly from the dynamical field equations. Hence, Eqs. (16) through (20) are rewritten to include parentheses around the indeterminate first partial derivatives on the wave surface $\Phi(x, z, t)=0$ :

$$
\rho\left(\frac{\partial U}{\partial x}\right) \frac{d x}{d t}+\rho\left(\frac{\partial U}{\partial z}\right) \frac{d z}{d t}+\left(\frac{\partial \sigma_{x x}}{\partial x}\right)+\left(\frac{\partial \sigma_{z x}}{\partial z}\right)=\rho \frac{d \dot{U}}{d t}
$$

$$
\rho\left(\frac{\partial W}{\partial x}\right) \frac{d x}{d t}+\rho\left(\frac{\partial W}{\partial z}\right) \frac{d z}{d t}+\left(\frac{\partial \sigma_{x z}}{\partial x}\right)+\left(\frac{\partial \sigma_{z z}}{\partial z}\right)=\rho \frac{d W}{d t}
$$

$$
\begin{gathered}
\left(\frac{\partial \boldsymbol{\sigma}_{z x}}{\partial x}\right) \frac{d x}{d t}+\left(\frac{\partial \boldsymbol{\sigma}_{z x}}{\partial z}\right) \frac{d z}{d t}+\mu\left(\frac{\partial W}{\partial x}\right)+\mu\left(\frac{\partial U}{\partial z}\right)=\frac{d \boldsymbol{\sigma}_{z x}}{d t} \\
\left(\frac{\partial \sigma_{z z}}{\partial x}\right) \frac{d x}{d t}+\left(\frac{\partial \sigma_{z z}}{\partial z}\right) \frac{d z}{d t}+\lambda\left(\frac{\partial U}{\partial x}\right) \\
+\lambda\left(\frac{\partial W}{\partial z}\right)+2 \mu\left(\frac{\partial W}{\partial z}\right)=\frac{d \sigma_{z z}}{d t} \\
\left(\frac{\partial \sigma_{x x}}{\partial x}\right) \frac{d x}{d t}+\left(\frac{\partial \sigma_{x x}}{\partial z}\right) \frac{d z}{d t}+\lambda\left(\frac{\partial U}{\partial x}\right) \\
+\lambda\left(\frac{\partial W}{\partial z}\right)+2 \mu\left(\frac{\partial U}{\partial x}\right)=\frac{d \sigma_{x x}}{d t}
\end{gathered}
$$

It can be seen from Eqs. (21) through (25) that the conventional method used to derive the characteristic equations is not applicable in this case, since the number of the indeterminate first partial derivatives exceeds the number of available equations. Therefore, rather than a direct treatment of Eqs. (21) through (25), which implies a direct dynamical analysis, it is first desired to confine the analysis to the kinematical conditions for the indeterminate first partial derivatives across the wave surface.

In his work on surfaces of discontinuity Hadamard developed kinematical conditions, which are also known as discontinuity relations, for the dependent variables and their derivatives across this surface. These analytical tools, based on Hadamard, are adapted here to formulate the proposed wave surface model. Corresponding to the basic conditions imposed on the surface of discontinuity, 
the relevant Hadamard's discontinuity relations (Ref. 7) are written as follows:

$$
\begin{aligned}
& {\left[\sigma_{i j, j}\right]=\delta_{i j} n_{j} \quad\left[\frac{\partial W}{\partial z}\right]=\delta_{z} n_{z}} \\
& {\left[\frac{\partial U}{\partial x}\right]=\delta_{x} n_{x}} \\
& {\left[\frac{\partial U}{\partial z}\right]=\delta_{x} n_{z}} \\
& {\left[\frac{\partial W}{\partial x}\right]=\delta_{z} n_{x}}
\end{aligned}
$$

where, if $f$ stands for any of the dependent variables (Fig. 2), then $[f, i]$ denotes the discontinuity in the first partial derivative across the wave surface $\Phi(x, z, t)=0$. The quantity $[f, i]$ is defined (Ref. 9) to be the value of the first partial derivative on the rear of the wave surface minus its value on the front of the wave surface, i.e.,

$$
[f, i]=f, i\left|\begin{array}{l}
\text { value of } f, i \\
\text { on rear of } \$
\end{array} \quad-f, i\right| \begin{gathered}
\text { value of } f, i \\
\text { on front of } \$
\end{gathered}
$$

$\delta_{i j}, \delta_{z}, \delta_{z}$ are arbitrary functions related to $\sigma_{i j}, U$, and $W$, respectively, and $n_{x}$ and $n_{z}$ are the Cartesian components of the unit normal vector $n$ to the wave surface $\Phi$.

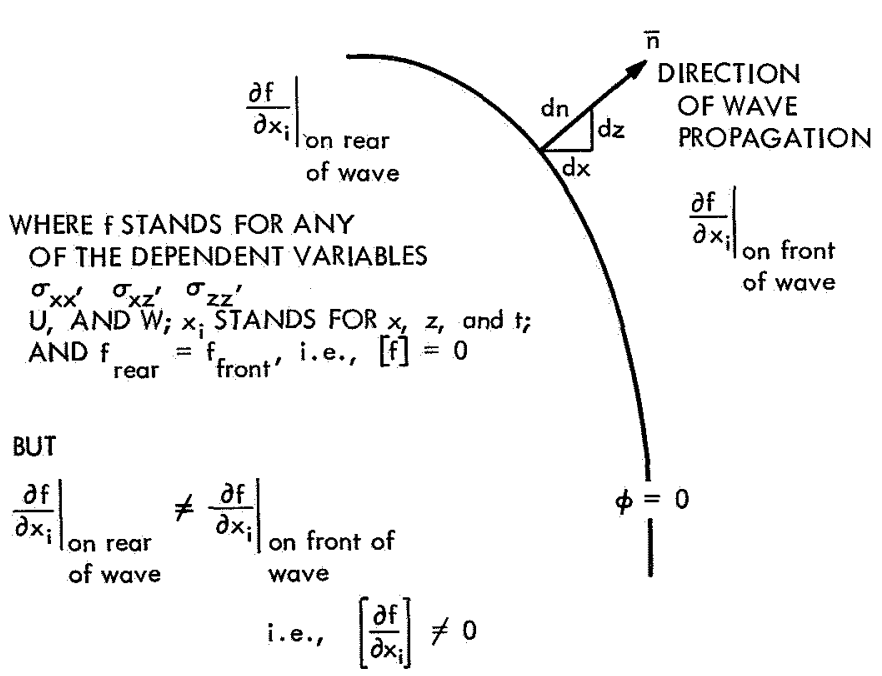

Fig. 2. Wave as a surface of discontinuity
The derivation of the discontinuity relations expressed in Eq. (26) is presented here for a discontinuity $[f, i]$, as follows: It is assumed that the function $f$ is continuous everywhere, but differentiable only in regions which are in the rear and in the front of the wave surface. In other words, discontinuities may occur in the first partial derivatives of the function $f$ across the wave surface $\Phi$. Two adjacent material particles on the wave surface $\Phi=0$ are considered in Fig. 3. The following relations may be written for the change in $f$ :

$$
\begin{array}{r}
d f_{\mathrm{I}}=f_{\mathrm{I}}\left(P^{\prime}\right)-f_{\mathrm{I}}(P)=f_{\mathrm{I}, i} d x_{i} \\
d f_{\mathrm{II}}=f_{\mathrm{II}}\left(P^{\prime}\right)-f_{\mathrm{II}}(P)=f_{\mathrm{II}, i} d x_{i}
\end{array}
$$

where $I=$ the region in front of the wave surface, $I I=$ the region in the rear of the surface, and the double indices imply summation. The relations are valid, since the change in $f$ considered here is with respect to spatial coordinates only. Equation (28), subtracted from Eq. (29), yields

$$
\left[f_{\mathrm{II}}\left(P^{\prime}\right)-f_{\mathrm{I}}\left(P^{\prime}\right)\right]-\left[f_{\mathrm{II}}(P)-f_{\mathrm{I}}(P)\right]=\left(f_{\mathrm{II}, i}-f_{\mathrm{I}, i}\right) d x_{i}
$$

but since it is assumed here that $f$ is continuous across the surface, or $[f]=f_{\mathrm{II}}-f_{\mathrm{I}}=0$, Eq. (30) now becomes

$$
\left(f_{\mathrm{II}, i}-f_{\mathrm{X}, i}\right) d x_{i}=0
$$

or, according to definition (27), Eq. (31) may be expressed as

$$
\left[f_{, i}\right] d x_{i}=0
$$

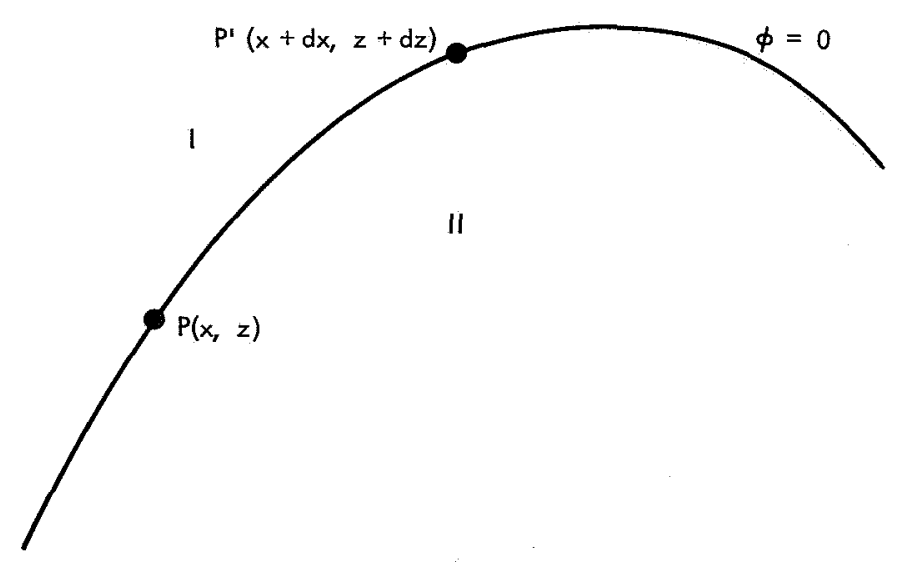

Fig. 3. Surface of discontinuity 
Furthermore, the wave surface equation at this instant of time is $\Phi\left(x_{i}\right)=0$ and thus,

$$
\Phi, i d x_{i}=d \Phi^{9}
$$

In view of Eqs. (32) and (33),

$$
\left[f_{, i}\right]=\xi \Phi, i
$$

where $\xi=$ a scalar of proportionality. Multiplication and division of this equation by the norm of the surface $\Phi$, i.e., by $\sqrt{\Phi_{, i} \Phi_{, i}}$ (where the double indices mean summation), yields:

$$
[f, i]=\xi \sqrt{\Phi, j} \Phi, \frac{\Phi, i}{\sqrt{\Phi_{, k} \Phi_{, k}}}
$$

By letting

$$
\xi \sqrt{\Phi, i} \Phi, i=8
$$

( $\delta$ is another scalar), the following discontinuity relation of Hadamard is obtained:

$$
[f, i]=\delta n_{i}
$$

Equation (35) was used to present Eq. (26), where $\boldsymbol{n}_{\boldsymbol{i}}$ are the components of a unit vector normal to the surface $\Phi=0$ (Ref. 9), i.e.,

$$
n_{i}=\frac{\Phi_{, i}}{\sqrt{\Phi, j \Phi, j}}
$$

or

$$
\begin{aligned}
& n_{x}=\frac{\frac{\partial \Phi}{\partial x}}{\sqrt{\left(\frac{\partial \Phi}{\partial x}\right)^{2}+\left(\frac{\partial \Phi}{\partial z}\right)^{2}}} \\
& n_{z}=\frac{\frac{\partial \Phi}{\partial z}}{\sqrt{\left(\frac{\partial \Phi}{\partial x}\right)^{2}+\left(\frac{\partial \Phi}{\partial z}\right)^{2}}}
\end{aligned}
$$

such that

$$
n_{x}^{2}+n_{z}^{2}=1
$$

letting

$$
\begin{aligned}
& n_{x}=\cos (n, x) \\
& n_{z}=\sin (n, x)
\end{aligned}
$$

To summarize, a wave surface is considered across which discontinuities may occur in the first partial derivatives of the dependent variables. For this wave surface model, discontinuity relations, based on the work of Hadamard, are provided. These discontinuity relations must now be incorporated into the dynamical field equations so that the dynamical conditions for the indeterminate first partial derivatives can be obtained. This is done in Section IV, where a transformation is used for this purpose.

\section{Dynamical Conditions}

Dynamical conditions for the indeterminate first partial derivatives are derived in this section. To present these conditions as equations which relate the indeterminate first partial derivatives in a compatible manner, a transformation is used by which the discontinuity relations are applied to the dynamical field equations.

The discontinuity relations expressed in Eqs. (26) or Eq. (35) are now transformed to a form in which first partial derivatives appear with respect to the normal of the wave surface. This is done in the following manner: The dependent variable $f=f\left(x, z, t_{0}\right)$ (at some instant of time $t_{0}$ ) in the discontinuity equation (35) is now considered to be a function of the normal to the wave surface, i.e., $f=f\left(x, z, t_{0}\right)$, where $x=x(n)$ and $z=z(n)$. and where $n$ is the distance measured along the normal to the wave surface $\Phi$. Thus, the composite function $f\left(x, z, t_{0}\right)=f\left[x(n), z(n), t_{0}\right]$ exists across the wave surface. In Cartesian tensor notation, it follows that

$$
\left[\frac{\partial f}{\partial n}\right]=\left[f_{, i}\right] \frac{d x_{i}}{d n}
$$

or

$$
\left[\frac{\partial f}{\partial n}\right]=\left[f_{, i}\right] n_{i}
$$

where it can be seen from Fig. 2 that $n_{i}=d x_{i} / d n$. Now, when both sides of Eq. (35) are multiplied by $n_{i}$ as follows,

$$
\left[f_{, i}\right] n_{i}=\delta n_{i} n_{i}
$$

$\left(n_{i} n_{i}=1\right.$ from Eq. 38), then 
In view of this last expression, Eq. (39) may be rewritten as

$$
\delta=\left[\frac{\partial f}{\partial n}\right]
$$

Consequently, Eq. (35) is rewritten in the following form:

$$
\left[f_{, i}\right]=\left[\frac{\partial f}{\partial n}\right] n_{i}
$$

or, by definition (27), Eq. (40) can further be written as

$$
f,\left.i\right|_{\substack{\text { value of } f, i \\
\text { on rear of } \$}}=\left[\frac{\partial f}{\partial n}\right] n_{i}+f,\left.i\right|_{\begin{array}{l}
\text { value of } f, i \\
\text { on front of } \Phi
\end{array}}
$$

where the value of $f_{, i}$ in front of the wave is finite and known from prescribed conditions. This is not the case, however, for the $f_{, i}$ on the rear of the wave surface. The first partial derivatives on the rear of the wave surface are created as the surface is being formed by the intersection of its tangent elements. Therefore, the $f_{, i}$ on the rear of the wave surface is considered here to be indeterminate. In accordance with the notation for the indeterminate first partial derivatives used in Eqs. (21) through (25), the above is rewritten as follows:

$$
(f, i)=\left[\frac{\partial f}{\partial n}\right] n_{i}+f, i
$$

Hence, in view of relation (41), and since $f$ stands for any of the dependent variables $\sigma_{i j}, U$, and $W$, the following relations for the indeterminate first partial derivatives on the wave surface are written:

$$
\left.\begin{array}{l}
\left(\frac{\partial U}{\partial x}\right)=\left[\frac{\partial U}{\partial n}\right] n_{x}+\frac{\partial U}{\partial x} \\
\left(\frac{\partial U}{\partial z}\right)=\left[\frac{\partial U}{\partial n}\right] n_{z}+\frac{\partial U}{\partial z} \\
\left(\frac{\partial W}{\partial z}\right)=\left[\frac{\partial W}{\partial n}\right] n_{z}+\frac{\partial W}{\partial z} \\
\left(\frac{\partial W}{\partial x}\right)=\left[\frac{\partial W}{\partial n}\right] n_{x}+\frac{\partial W}{\partial x} \\
\left(\frac{\partial \sigma_{x x}}{\partial x}\right)=\left[\frac{\partial \sigma_{x x}}{\partial n}\right] n_{z}+\frac{\partial \sigma_{x x}}{\partial x} \\
\left(\frac{\partial \sigma_{x x}}{\partial z}\right)=\left[\frac{\partial \sigma_{x x}}{\partial n}\right] n_{z}+\frac{\partial \sigma_{x x}}{\partial z} \\
\left(\frac{\partial \sigma_{x z}}{\partial x}\right)=\left[\frac{\partial \sigma_{x z}}{\partial n}\right] n_{x}+\frac{\partial \sigma_{x z}}{\partial x} \\
\left(\frac{\partial \sigma_{x z}}{\partial z}\right)=\left[\frac{\partial \sigma_{x z}}{\partial n}\right] n_{z}+\frac{\partial \sigma_{x z}}{\partial z} \\
\left(\frac{\partial \sigma_{z z}}{\partial x}\right)=\left[\frac{\partial \sigma_{z z}}{\partial n}\right] n_{x}+\frac{\partial \sigma_{z z}}{\partial x} \\
\left(\frac{\partial \sigma_{z z}}{\partial z}\right)=\left[\frac{\partial \sigma_{z z}}{\partial n}\right] n_{z}+\frac{\partial \sigma_{z z}}{\partial z}
\end{array}\right\}
$$

The analysis done so far has been confined to kinematical conditions for the indeterminate first partial derivatives of the dependent variables on the wave surface. These kinematical conditions, expressed in Eqs. (42), are now applied to dynamical field equations (21) through (25) to yield the following relations:

$$
\left.\begin{array}{c}
\rho\left[\frac{\partial U}{\partial n}\right]\left(n_{x} \frac{d x}{d t}+n_{z} \frac{d z}{d t}\right)+\left[\frac{\partial \sigma_{x x}}{\partial n}\right] n_{x}+\left[\frac{\partial \sigma_{z x}}{\partial n}\right] n_{z}=R_{U} \\
\rho\left[\frac{\partial W}{\partial n}\right]\left(n_{x} \frac{d x}{d t}+n_{z} \frac{d z}{d t}\right)+\left[\frac{\partial \sigma_{z x}}{\partial n}\right] n_{x}+\left[\frac{\partial \sigma_{z z}}{\partial n}\right] n_{z}=R_{W} \\
{\left[\frac{\partial \sigma_{x z}}{\partial n}\right]\left(n_{x} \frac{d x}{d t}+n_{z} \frac{d z}{d t}\right)+\mu\left[\frac{\partial W}{\partial n}\right] n_{x}+\mu\left[\frac{\partial U}{\partial n}\right] n_{z}=R_{x z}} \\
{\left[\frac{\partial \sigma_{z z}}{\partial n}\right]\left(n_{x} \frac{d x}{d t}+n_{z} \frac{d z}{d t}\right)+\lambda\left[\frac{\partial U}{\partial n}\right] n_{x}+(\lambda+2 \mu)\left[\frac{\partial W}{\partial n}\right] n_{z}=R_{z z}} \\
{\left[\frac{\partial \sigma_{x x}}{\partial n}\right]\left(n_{x} \frac{d x}{d t}+n_{z} \frac{d z}{d t}\right)+\lambda\left[\frac{\partial W}{\partial n}\right] n_{z}+(\lambda+2 \mu)\left[\frac{\partial U}{\partial n}\right] n_{x}=R_{x x}}
\end{array}\right\}
$$


where

$$
\left.\begin{array}{l}
\boldsymbol{R}_{U}=\rho \frac{d U}{d t}-\rho\left(n_{x} \frac{d x}{d t}+n_{z} \frac{d z}{d t}\right)\left(\frac{\partial U}{\partial x} n_{x}+\frac{\partial U}{\partial z} n_{z}\right)-\frac{\partial \sigma_{x x}}{\partial x}-\frac{\partial \sigma_{x z}}{\partial z} \\
\boldsymbol{R}_{W}=\rho \frac{d W}{d t}-\rho\left(n_{x} \frac{d x}{d t}+n_{z} \frac{d z}{d t}\right)\left(\frac{\partial W}{\partial x} n_{x}+\frac{\partial W}{\partial z} n_{z}\right)-\frac{\partial \sigma_{z z}}{\partial z}-\frac{\partial \sigma_{x z}}{\partial x} \\
R_{x z}=\frac{d \sigma_{x z}}{d t}-\left(n_{x} \frac{d x}{d t}+n_{z} \frac{d z}{d t}\right)\left(\frac{\partial \sigma_{x z}}{\partial x} n_{x}+\frac{\partial \sigma_{x z}}{\partial z} n_{z}\right)-\mu\left(\frac{\partial W}{\partial x}+\frac{\partial U}{\partial z}\right) \\
\boldsymbol{R}_{z z}=\frac{d \sigma_{z z}}{d t}-\left(n_{x} \frac{d x}{d t}+n_{z} \frac{d z}{d t}\right)\left(\frac{\partial \sigma_{z z}}{\partial x} n_{x}+\frac{\partial \sigma_{z z}}{\partial z} n_{z}\right)-(\lambda+2 \mu) \frac{\partial W}{\partial z}-\lambda \frac{\partial U}{\partial x} \\
R_{x x}=\frac{d \sigma_{x x}}{d t}-\left(n_{x} \frac{d x}{d t}+n_{z} \frac{d z}{d t}\right)\left(\frac{\partial \sigma_{x x}}{\partial x} n_{x}+\frac{\partial \sigma_{x x}}{\partial z} n_{z}\right)-(\lambda+2 \mu) \frac{\partial U}{\partial x}-\lambda \frac{\partial W}{\partial z}
\end{array}\right\}
$$

Let

$$
G=n_{x} \frac{d x}{d t}+n_{z} \frac{d z}{d t}
$$

which is recognized as the common term in the system of Eqs. (43) and (44). Therefore, Eqs. (43) become

$$
\left.\begin{array}{r}
\rho G\left[\frac{\partial U}{\partial n}\right]+\left[\frac{\partial \boldsymbol{\sigma}_{x x}}{\partial n}\right] n_{x}+\left[\frac{\partial \boldsymbol{\sigma}_{z x}}{\partial n}\right] n_{z}=R_{U} \\
\rho G\left[\frac{\partial W}{\partial n}\right]+\left[\frac{\partial \boldsymbol{\sigma}_{z x}}{\partial n}\right] n_{x}+\left[\frac{\partial \boldsymbol{\sigma}_{z z}}{\partial n}\right] n_{z}=\boldsymbol{R}_{W} \\
G\left[\frac{\partial \boldsymbol{\sigma}_{z x}}{\partial n}\right]+\mu\left[\frac{\partial W}{\partial n}\right] n_{x}+\mu\left[\frac{\partial U}{\partial n}\right] n_{z}=\boldsymbol{R}_{x z} \\
G\left[\frac{\partial \boldsymbol{\sigma}_{z z}}{\partial \boldsymbol{n}}\right]+\lambda\left[\frac{\partial \boldsymbol{U}}{\partial n}\right] n_{x}+(\lambda+2 \mu)\left[\frac{\partial W}{\partial n}\right] n_{z}=\boldsymbol{R}_{z z} \\
G\left[\frac{\partial \boldsymbol{\sigma}_{x x}}{\partial \boldsymbol{n}}\right]+\lambda\left[\frac{\partial W}{\partial n}\right] n_{z}+(\lambda+2 \mu)\left[\frac{\partial U}{\partial n}\right] n_{x}=\boldsymbol{R}_{x x}
\end{array}\right\}
$$

Rewriting Eqs. (46) in matrix form gives

$\left[\begin{array}{ccccc}\rho G & 0 & n_{x} & 0 & n_{z} \\ 0 & \rho G & 0 & n_{z} & n_{x} \\ \mu n_{z} & \mu n_{x} & 0 & 0 & G \\ \lambda n_{x} & (\lambda+2 \mu) n_{z} & 0 & G & 0 \\ (\lambda+2 \mu) n_{x} & \lambda n_{z} & G & 0 & 0\end{array}\right]\left\{\begin{array}{l}{\left[\frac{\partial U}{\partial n}\right]} \\ {\left[\frac{\partial W}{\partial n}\right]} \\ {\left[\frac{\partial \sigma_{x x}}{\partial n}\right]} \\ {\left[\frac{\partial \sigma_{z z}}{\partial n}\right]} \\ {\left[\frac{\partial \sigma_{x z}}{\partial n}\right]}\end{array}\right\}\left\{\begin{array}{c}R_{U} \\ R_{W} \\ R_{x z} \\ R_{z z} \\ R_{x x}\end{array}\right\}$


Hence, Eqs. (47) present a system of five equations which relate five first partial derivatives

$$
\left[\frac{\partial U}{\partial n}\right],\left[\frac{\partial W}{\partial n}\right],\left[\frac{\partial \sigma_{x x}}{\partial n}\right],\left[\frac{\partial \sigma_{z z}}{\partial n}\right], \text { and }\left[\frac{\partial \sigma_{z x}}{\partial n}\right]
$$

of the five dependent variables $U, W, \sigma_{x x}, \sigma_{z z}$, and $\boldsymbol{\sigma}_{x z}$. The system of differential equations (47) is then denoted as the dynamical conditions, since it is from these relations that the wave surfaces and the characteristic equations will be derived.

In order to derive the wave surfaces and then the characteristic equations which hold on these surfaces from the dynamical conditions, the following conventional approach (Ref. 3) is attempted. To solve any of the unknowns, say $[\partial U / \partial n]$, in Eq. (47), a ratio is needed whose denominator is the determinant of the coefficients and whose numerator is the determinant, where the determinant's first column is replaced by the right-hand column of Eq. (47). However, since it is required that $[\partial U / \partial n]$ be discontinuous, the denominator is made to vanish. Furthermore, since it was established that this discontinuity takes place across the wave surface, it can be concluded that by equating the denominator to zero, the wave surface can be determined. Therefore, the determinant of the coefficients when equated to zero is denoted as the characteristic condition. On the other hand, it is also required that the discontinuities be related in order to define the tangent elements (see Section II) of the wave surface. This relatedness is established when the numerator is also made to vanish. The numerator, which is equated to zero, and the equations for the characteristic surfaces will yield the characteristic equations.

In view of the above description of the dynamical conditions, steps are taken to determine (1) the wave surface (or surfaces) from the characteristic condition and the generators of the surfaces, which are the bicharacteristic curves (Section V) and (2) the characteristic equation (or equations) which hold along the bicharacteristic curves (Section VI).

\section{Characteristic Condition and Bicharacteristic Curves}

Once the dynamical conditions for the indeterminate first partial derivatives have been established, the wave surfaces can be determined from the characteristic condition. The bicharacteristic curves are then derived from these characteristic surfaces.
The determinant of the coefficients of matrix (47) is equated to zero in order to establish the characteristic condition, i.e.,

$$
\left|\begin{array}{ccccc}
\rho G & 0 & n_{x} & 0 & n_{z} \\
0 & \rho G & 0 & n_{z} & n_{x} \\
\mu n_{z} & \mu n_{x} & 0 & 0 & G \\
\lambda n_{x} & (\lambda+2 \mu) n_{z} & 0 & G & 0 \\
(\lambda+2 \mu) n_{x} & \lambda n_{z} & G & 0 & 0
\end{array}\right|=0
$$

The determinant is expanded to yield the following equation:

$$
G\left[G^{4}-\frac{\lambda+3 \mu}{\rho} G^{2}+\frac{\mu(\lambda+2 \mu)}{\rho^{2}}\right]=0
$$

The solution to this equation yields five distinct and real roots:

$$
\begin{gathered}
G_{1}=\sqrt{\frac{\lambda+2 \mu}{\rho}} \\
G_{2}=-\sqrt{\frac{\lambda+2 \mu}{\rho}} \\
G_{3}=\sqrt{\frac{\mu}{\rho}} \\
G_{4}=-\sqrt{\frac{\mu}{\rho}} \\
G_{5}=0
\end{gathered}
$$

In order to interpret the meaning of these roots, a wave velocity $g$ is defined as follows: Let $\Phi$ be the wave surface, and let a material particle $P$ be on this surface at time $t$. At time $t+d t$, the normal $n$ at $P$ to $\Phi(t)$ will intersect the same wave surface at a different material particle $P^{\prime}$ (Fig. 4). Hence, the velocity $g$ of the wave surface $\Phi$ at $P$ at the instant $t$ is defined to be

$$
\mathrm{g}=\frac{\overrightarrow{P P^{\prime}}}{d t}
$$




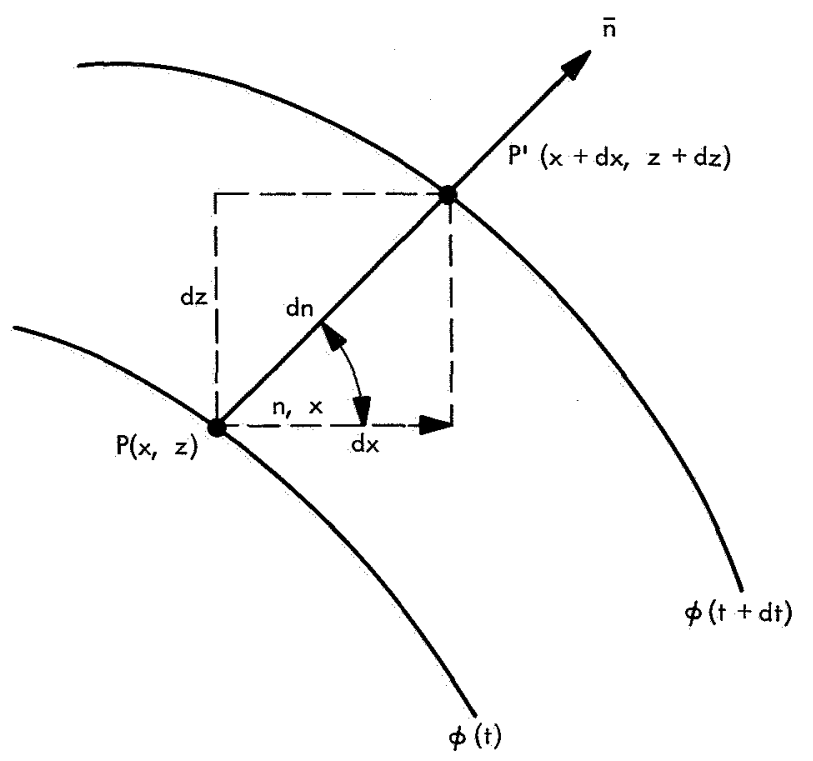

Fig. 4. Wave surface

or the wave surface speed is

$$
g=\frac{d n}{d t}
$$

where it is apparent from Fig. 4 and Eq. (38) that $d n$ may be presented by vector addition as

$$
d n=n_{x} d x+n_{z} d z
$$

or

$$
g=n_{x} \frac{d x}{d t}+n_{z} \frac{d z}{d t}
$$

This expression is identical to the relation expressed in Eq. (45). Therefore, it is clear that $G$ is identical to $g$, which is the propagation speed of the wave surface. This is also consistent with the dimensions of $G$ (Eqs. 50 through 53). Furthermore, from Fig. 4, it is also apparent that

$$
(d n)^{2}=(d x)^{2}+(d z)^{2}
$$

or, in view of definition (55), that

$$
(d x)^{2}+(d z)^{2}=G^{2}(d t)^{2}
$$

Equation (57) defines the Monge cone (Ref. 10) (see Figs. 1 and 5). Each wave surface through the apex of this cone touches the cone along the bicharacteristic curves. The bicharacteristic curves are easily found by

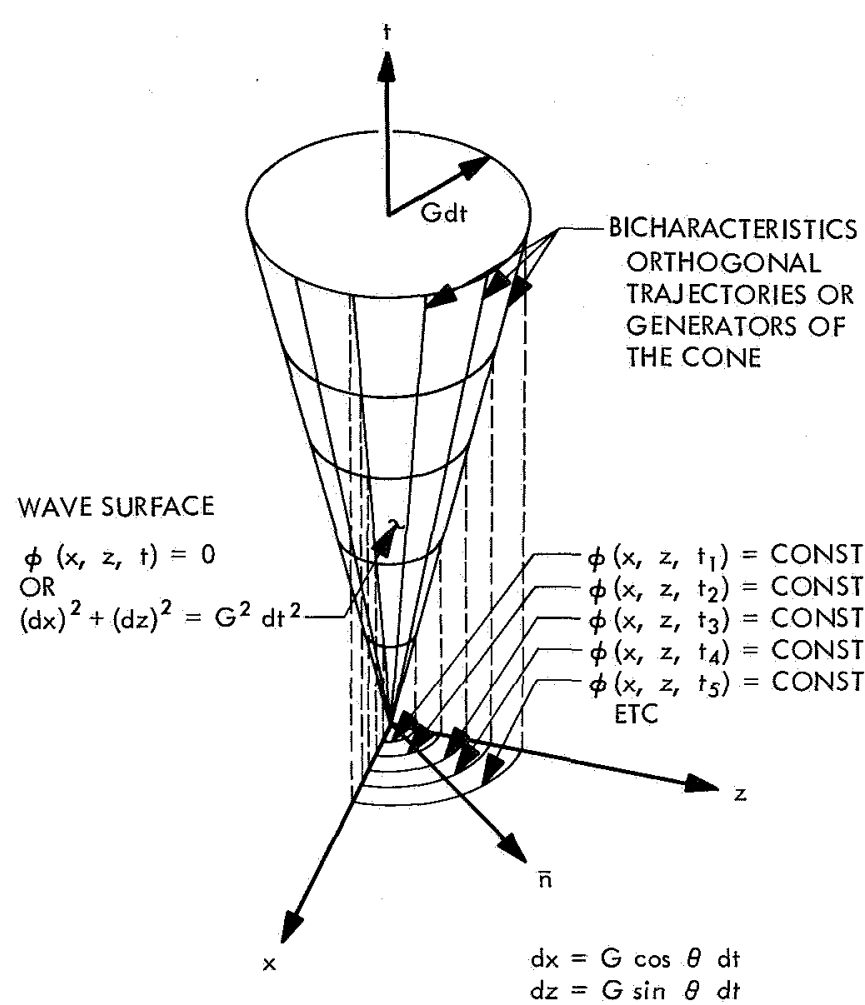

THE BICHARACTERISTICS ARE FORMED BY THE INTERSECTION OF THE SURFACE $\phi(x, z, t)=0$ WITH THE SHEAF OF PLANES PASSING THROUGH THE T-AXIS

Fig. 5. Typical wave surface

examining Eq. (57) in Fig. 6, and may be presented as follows:

$$
\begin{aligned}
& d x=G \cos \theta d t \\
& d z=G \sin \theta d t
\end{aligned}
$$

or

$$
\begin{aligned}
& d x=G n_{x} d t \\
& d z=G n_{z} d t
\end{aligned}
$$

The next task is to derive the characteristic equations which relate the dependent variables along these bicharacteristic curves. Before this is done, however, some conclusions should be reached concerning the dynamical nature of the obtained wave speeds as expressed in Eqs. (50) through (54).

First, the nature of Eq. (54) (i.e., $G_{5}=0$ ) will be examined. For this purpose, the equations of motion, 


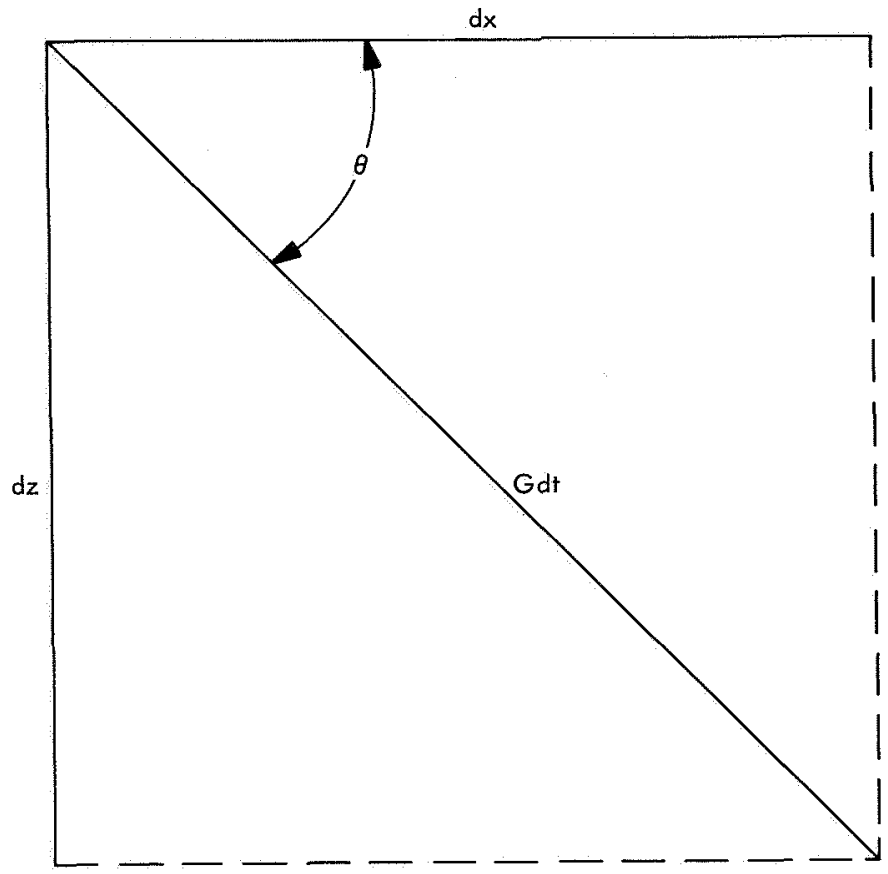

Fig. 6. Representation of Eq. (57)

Eqs. (16) and (17), are rewritten for the wave surface, in which case they include the discontinuity notations and the relations $d x / d t=G n_{x}$ and $d z / d t=G n_{z}$ (see Fig. 4).

$\left[\frac{\partial \sigma_{x x}}{\partial x}\right]+\left[\frac{\partial \sigma_{x z}}{\partial z}\right]=-\rho G\left[\frac{\partial U}{\partial x}\right] n_{x}-\rho G\left[\frac{\partial U}{\partial z}\right] n_{z}+\rho\left[\frac{d U}{d t}\right]$

$\left[\frac{\partial \boldsymbol{\sigma}_{x z}}{\partial x}\right]+\left[\frac{\partial \sigma_{z z}}{\partial z}\right]=-\rho G\left[\frac{\partial W}{\partial x}\right] n_{x}-\rho G\left[\frac{\partial W}{\partial z}\right] n_{z}+\rho\left[\frac{d W}{d t}\right]$

Obviously, since $U$ and $W$ are assumed to be continuous across the wave surface,

$$
\left[\frac{d U}{d t}\right]=\left[\frac{d W}{d t}\right]=0
$$

When zero is substituted for $G$ in the above equations, it can be seen that this root represents a front where static deformation takes place.

In order to interpret the significance of the roots $G^{2}=(\lambda+2 \mu) / \rho$ and $G^{2}=\mu / \rho$, the discontinuity relations (26) are applied to Eqs. (16) through (20), which are combined to yield the following two relations:

$$
\left.\begin{array}{l}
n_{x}(\lambda+\mu)\left(n_{x} \delta_{x}+n_{z} \delta_{z}\right)=\left(\rho G^{2}-\mu\right) \delta_{x} \\
n_{z}(\lambda+\mu) \cdot\left(n_{x} \delta_{x}+n_{z} \delta_{z}\right)=\left(\rho G^{2}-\mu\right) \delta_{z}
\end{array}\right\}
$$

When $\lambda+2 \mu / \rho$ is substituted for $G^{2}$ in Eqs. (59), the following features are obtained:

$$
\left.\begin{array}{l}
n_{x}\left(n_{x} \delta_{x}+n_{z} \delta_{z}\right)=\delta_{x} \\
n_{z}\left(n_{z} \delta_{z}+n_{x} \delta_{x}\right)=\delta_{z}
\end{array}\right\}
$$

These relations imply that the vector $\delta$ is parallel to vector $\mathbf{n}$, or that the material particle at the wave surface moves parallel to the propagating wave. Also, the linearized vorticity at the wave surface is

$$
1 / 2\left(\left[\frac{\partial U}{\partial z}\right]-\left[\frac{\partial W}{\partial x}\right]\right)
$$

In view of Eqs. (26),

$$
\left[\frac{\partial U}{\partial z}\right]-\left[\frac{\partial W}{\partial x}\right]=\delta_{x} n_{z}-\delta_{z} n_{x}
$$

but as a result of the relations (60), the vorticity at the wave surface vanishes. A wave characterized by these features is known as the longitudinal wave, which propagates with speeds expressed in Eqs. (50) and (51); $G_{1}$ and $G_{2}$ are now replaced by the notations $G_{L}^{+}$and $G_{L}^{-}$, respectively, where the subscript $L$ refers to longitudinal waves. This observation, as well as the following one, is a restatement of T. Y. Thomas' observation (Ref. 11).

The substitution of $\mu / \rho$ into Eq. (53) yields the following relation:

$$
n_{x} \delta_{x}+n_{z} \delta_{z}=0
$$

which implies that

$$
\frac{\partial U}{\partial x}+\frac{\partial W}{\partial z}=0
$$

Thus, the dilatation at the wave vanishes. Also, on the basis of relations (59), the term $n_{x} \delta_{x}+n_{z} \delta_{z}$ represents a scalar, which may be written as a dot product. In conjunction with relation (61), this term becomes $n \cdot \delta=0$. A wave consisting of these features is known as the shear wave, which propagates with the speeds expressed in Eqs. (52) and (53). The speeds $G_{3}$ and $G_{4}$ are now replaced by the notations $G_{S}^{+}$and $G_{\bar{S}}$, respectively, where the subscript $S$ refers to shear waves.

Thus far, the wave surfaces, their speeds of propagation, and their bicharacteristic curves have been established. In Section VI, the characteristic equations which hold along the bicharacteristic curves and on the wave surfaces will be derived. 


\section{Carfesian Characteristic Equations}

In this section, the characteristic equations are derived from the dynamical conditions. As was described in Section IV, the procedure used to obtain the characteristic equations is as follows: Each column in the coefficient determinant of Eq. (48) is replaced consecutively by the column on the right-hand side. The determinant is then made to vanish for each such replacement. The expansion of the determinant yields the desired relation. To demonstrate this operation, for example for $[\partial U / \partial n]$, it follows that

$$
\left|\begin{array}{ccccc}
\boldsymbol{R}_{U} & 0 & n_{x} & 0 & n_{z} \\
\boldsymbol{R}_{W} & \rho G & \mathbf{0} & n_{z} & n_{x} \\
\boldsymbol{R}_{x z} & \mu n_{x} & 0 & 0 & G \\
\boldsymbol{R}_{z z} & (\lambda+2 \mu) n_{z} & 0 & \boldsymbol{G} & 0 \\
\boldsymbol{R}_{x x} & \lambda n_{z} & \mathrm{G} & \mathbf{0} & 0
\end{array}\right|=\mathbf{0}
$$

For $\left[\partial \sigma_{z x} / \partial n\right]$,

$$
\begin{aligned}
R_{z z} \mu n_{x} n_{z}[ & \left.-\rho G^{2}-\lambda n_{z}^{2}+(\lambda+2 \mu) n_{x}^{2}\right] \\
& -R_{W} G_{\mu} n_{x}\left[-\rho G^{2}-\lambda n_{z}^{2}+(\lambda+2 \mu) n_{x}^{2}\right] \\
& +R_{x x} \mu n_{x} n_{z}\left[-\rho G^{2}-\lambda n_{x}^{2}+(\lambda+2 \mu) n_{z}^{2}\right] \\
& -R_{U} G \mu n_{z}\left[-\rho G^{2}-\lambda n_{x}^{2}+(\lambda+2 \mu) n_{z}^{2}\right] \\
& +R_{x z}\left\{\rho G^{2}\left[-\rho G^{2}+(\lambda+2 \mu)\right]\right. \\
& \left.-4 \mu(\lambda+\mu) n_{x}^{2} n_{z}^{2}\right\}=0
\end{aligned}
$$

Upon expansion of this determinant, the following relation is obtained for $[\partial U / \partial n]$ :

$$
\begin{aligned}
R_{z z}(\lambda & +\mu) n_{x} n_{z}^{2}-R_{W} G(\lambda+\mu) n_{x} n_{z} \\
& +R_{x x} n_{x}\left[\rho G^{2}-\mu-(\lambda+\mu) n_{z}^{2}\right] \\
& -R_{V} G\left[\rho G^{2}-\mu-(\lambda+\mu) n_{z}^{2}\right] \\
& +R_{x z} n_{z}\left[\rho G^{2}+\lambda n_{x}^{2}-(\lambda+2 \mu) n_{z}^{2}\right]=0
\end{aligned}
$$

The following equations are obtained similarly:

For $\left[\partial \sigma_{x x} / \partial n\right]$,

$$
\begin{aligned}
R_{z z} n_{z}^{2}[ & \left.-\rho G^{2} \lambda+\lambda \mu n_{z}^{2}-\mu(\lambda+2 \mu) n_{x}^{2}\right] \\
& -R_{W} G n_{z}\left[-\rho G^{2} \lambda+\lambda \mu n_{z}^{2}-\mu(\lambda+2 \mu) n_{x}^{2}\right] \\
& +R_{x x}\left\{\rho G^{2}\left[-\rho G^{2}+\mu+(\lambda+2 \mu) n_{z}^{2}\right]\right. \\
& \left.+\mu n_{z}^{2}\left[\lambda n_{x}^{2}-(\lambda+2 \mu) n_{z}^{2}\right]\right\} \\
& -R_{U} G n_{x}\left[(\lambda+2 \mu)\left(-\rho G^{2}+\mu\right)+2 \mu(\lambda+\mu) n_{z}^{2}\right] \\
& +2 R_{x z}(\lambda+\mu) n_{x} n_{z}\left(-\rho G^{2}+2 \mu n_{z}^{2}\right)=0
\end{aligned}
$$

The characteristic surfaces as expressed in relations (50) through (54) are substituted for $G$ in Eqs. (63) through (67). The result is five distinct characteristic equations which relate the five dependent variables $\sigma_{x x}, \sigma_{z z}, \sigma_{x z}, U$, and $W$ along the bicharacteristic curves expressed in Eq. (58). These characteristic equations are:

$$
n_{z}^{2} R_{z z}+n_{x}^{2} R_{x x}+2 n_{x} n_{z} R_{z x}-\mathrm{G}_{L}^{+}\left(n_{z} R_{W}+n_{x} R_{U}\right)=0
$$

along

$$
\begin{aligned}
& d x=G_{L}^{+} n_{x} d t \\
& d z=G_{L}^{+} n_{z} d t
\end{aligned}
$$

where

$$
\begin{gathered}
G_{L}^{+}=\sqrt{\frac{\lambda+2 \mu}{\rho}} \\
n_{z}^{2} R_{z z}+n_{x}^{2} R_{x x}+2 n_{x} n_{z} R_{x z}-G_{L}\left(n_{z} R_{W}+n_{x} R_{U}\right)=0
\end{gathered}
$$

along

$$
\begin{aligned}
& -R_{W} G\left[\rho G^{2}-\mu-(\lambda+\mu) n_{x}^{2}\right] \\
& +R_{x x}(\lambda+\mu) n_{x}^{2} n_{z}-R_{U} G(\lambda+\mu) n_{x} n_{z} \\
& +R_{z x} n_{x}\left[\rho G^{2}-(\lambda+2 \mu) n_{x}^{2}+\lambda n_{z}^{2}\right]=0
\end{aligned}
$$$$
\boldsymbol{R}_{z z} n_{z}\left[\rho G^{2}-\mu-(\lambda+\mu) n_{x}^{2}\right]
$$$$
-R_{W} G n_{z}\left[(\lambda+2 \mu)\left(-\rho G^{2}+\mu\right)+2 \mu(\lambda+\mu) n_{x}^{2}\right]
$$ 
where

$$
\begin{gathered}
G_{L}^{-}=-\sqrt{\frac{\lambda+2 \mu}{\rho}} \\
n_{m} n_{z}\left(R_{z z}-R_{x x}\right)+\left(1-2 n_{z}^{2}\right) R_{x z}-G_{S}^{+}\left(n_{x} R_{W}-n_{z} R_{U}\right)=0
\end{gathered}
$$

along

$$
\begin{aligned}
& d x=G_{s}^{+} n_{x} d t \\
& d z=G_{s}^{+} n_{z} d t
\end{aligned}
$$

where

$$
\begin{gathered}
G_{S}^{+}=\sqrt{\frac{\mu}{\rho}} \\
n_{x} n_{z}\left(R_{z z}-R_{x x}\right)+\left(1-2 n_{z}^{2}\right) R_{x z}-G_{s}^{-}\left(n_{x} R_{W}-n_{z} R_{U}\right)=0
\end{gathered}
$$

along

$$
\begin{aligned}
& d x=G_{S}^{-} n_{x} d t \\
& d z=G_{S}^{-} n_{z} d t
\end{aligned}
$$

where

$$
\begin{gathered}
G_{\bar{s}}^{-}=-\sqrt{\frac{\mu}{\rho}} \\
{\left[\lambda n_{x}^{2}-(\lambda+2 \mu) n_{z}^{2}\right] R_{x x}+\left[\lambda n_{z}^{2}-(\lambda+2 \mu) n_{x}^{2}\right] R_{z z}} \\
+4 n_{x} n_{z}(\lambda+\mu) R_{x z}=0
\end{gathered}
$$

for

$$
G=0
$$

along

$$
\begin{aligned}
& d x=0 \\
& d z=0
\end{aligned}
$$

where the $R$ are expressed in Eqs. (44) when

$$
n_{x} d x / d t+n_{z} d z / d t
$$

is replaced by the relevant $G$.

Boundary-value problems can now be solved by employing the characteristic equations (68) through (72), as will be demonstrated later. Section VII is devoted to the derivation of the characteristic equations in the cylindrical coordinate system.

\section{Cylindrical Characteristic Equations}

This section is presented in order to accommodate many practical problems in which the cylindrical coordinate system is advantageous. A system of five differential equations which relate the five unknown functions $\sigma_{r r}$, $\sigma_{\theta \theta}, \sigma_{r \theta}, U_{r}$, and $U_{\theta}$ along the bicharacteristic curves is to be derived here. The principles of the theory and the derivations are analogous to the analysis used to derive Eqs. (68) through (72), and reference should be made to the preceding sections.

The equations of motion for linear elastic material, where plane strain is prevalent, are

$$
\begin{gathered}
\frac{\partial \sigma_{r r}}{\partial r}+\frac{1}{r} \frac{\partial \sigma_{r \theta}}{\partial \theta}+\frac{\sigma_{r r}-\sigma_{\theta \theta}}{r}=\rho \frac{\partial^{2} u_{r}}{\partial t^{2}} \\
\frac{1}{r} \frac{\partial \sigma_{\theta \theta}}{\partial \theta}+\frac{\partial \sigma_{r \theta}}{\partial r}+\frac{2 \sigma_{r \theta}}{r}=\rho \frac{\partial^{2} u_{\theta}}{\partial t^{2}}
\end{gathered}
$$

where

$$
\rho=\text { density }
$$

$u_{r}$ and $u_{\theta}=$ radial and tangential displacements, respectively

$\sigma_{r r}, \sigma_{\theta \theta}$, and $\sigma_{r \theta}=$ stress components in the $r, \theta$-plane

$r$ and $\theta=$ plane cylindrical coordinates

$t=$ the time dimension

The stress-displacement relations are

$$
\sigma_{r r}=\lambda\left(\frac{\partial u_{r}}{\partial r}+\frac{u_{r}}{r}+\frac{1}{r} \frac{\partial u_{\theta}}{\partial \theta}\right)+2 \mu \frac{\partial u_{r}}{\theta r}
$$

$$
\sigma_{\theta \theta}=\lambda\left(\frac{\partial u_{r}}{\partial r}+\frac{u_{r}}{r}+\frac{1}{r} \frac{\partial u_{\theta}}{\partial \theta}\right)+2 \mu\left(\frac{u_{r}}{r}+\frac{1}{r} \frac{\partial u_{\theta}}{\partial \theta}\right)
$$

$$
\sigma_{r \theta}=\mu\left(\frac{\partial u_{\theta}}{\partial r}-\frac{u_{\theta}}{r}+\frac{1}{r} \frac{\partial u_{r}}{\partial \theta}\right)
$$

where $\mu$ and $\lambda=$ Lamé's constants. Let

$$
\begin{aligned}
& \frac{\partial u_{r}}{\partial t}=U_{r} \\
& \frac{\partial u_{\theta}}{\partial t}=U_{\theta}
\end{aligned}
$$


where $U_{r}$ and $U_{\theta}=$ the radial and tangential velocities, respectively, and, upon differentiation of Eqs. 75, 76, and 77 with respect to time, Eqs. (73) through (77) become

$$
\begin{gathered}
\frac{\partial \boldsymbol{\sigma}_{r r}}{\partial r}+\frac{1}{r} \frac{\partial \boldsymbol{\sigma}_{r \theta}}{\partial \theta}+\frac{\boldsymbol{\sigma}_{r r}-\boldsymbol{\sigma}_{\theta \theta}}{r}=\rho \frac{\partial U_{r}}{\partial t} \\
\frac{1}{r} \frac{\partial \sigma_{\theta \theta}}{\partial \theta}+\frac{\partial \sigma_{r \theta}}{\partial r}+\frac{2 \sigma_{r \theta}}{r}=\rho \frac{\partial U_{\theta}}{\partial t} \\
\frac{\partial \boldsymbol{\sigma}_{r r}}{\partial t}=\lambda\left(\frac{\partial U_{r}}{\partial r}+\frac{U_{r}}{r}+\frac{1}{r} \frac{\partial U_{\theta}}{\partial \theta}\right)+2 \mu \frac{\partial U_{r}}{\partial r} \\
\frac{\partial \sigma_{\theta \theta}}{\partial t}=\lambda\left(\frac{\partial U_{r}}{\partial r}+\frac{U_{r}}{r}+\frac{1}{r} \frac{\partial U_{\theta}}{\partial \theta}\right) \\
+2 \mu\left(\frac{U_{r}}{r}+\frac{1}{r} \frac{\partial U_{\theta}}{\partial \theta}\right) \\
\frac{\partial \sigma_{r \theta}}{\partial t}=\mu\left(\frac{\partial U_{\theta}}{\partial r}-\frac{U_{\theta}}{r}+\frac{1}{r} \frac{\partial U_{r}}{\partial \theta}\right)
\end{gathered}
$$

Also, the region in the physical space $(x, z, t)$ is considered where the five dependent variables are continuous and differentiable. For this region, the following relations exist:

$$
\begin{aligned}
& \frac{d U_{r}}{d t}=\frac{\partial U_{r}}{\partial r} \frac{d r}{d t}+\frac{\partial U_{r}}{\partial \theta} \frac{d \theta}{d t}+\frac{\partial U_{r}}{\partial t} \\
& \frac{d U_{\theta}}{d t}=\frac{\partial U_{\theta}}{\partial r} \frac{d r}{d t}+\frac{\partial U_{\theta}}{\partial \theta} \frac{d \theta}{d t}+\frac{\partial U_{\theta}}{\partial t} \\
& \frac{d \sigma_{r r}}{d t}=\frac{\partial \sigma_{r r}}{\partial r} \frac{d r}{d t}+\frac{\partial \sigma_{r r}}{\partial \theta} \frac{d \theta}{d t}+\frac{\partial \sigma_{r r}}{\partial t} \\
& \frac{d \sigma_{\theta \theta}}{d t}=\frac{\partial \sigma_{\theta \theta}}{\partial r} \frac{d r}{d t}+\frac{\partial \sigma_{\theta \theta}}{\partial \theta} \frac{d \theta}{d t}+\frac{\partial \sigma_{\theta \theta}}{\partial t} \\
& \frac{d \sigma_{r \theta}}{d t}=\frac{\partial \sigma_{r \theta}}{\partial r} \frac{d r}{d t}+\frac{\partial \sigma_{r \theta}}{\partial \theta} \frac{d \theta}{d t}+\frac{\partial \sigma_{r \theta}}{\partial t}
\end{aligned}
$$

By the same argument used to derive Eq. (41), it follows that

$$
\left.\begin{array}{l}
\left(\frac{\partial U_{r}}{\partial r}\right)=\left[\frac{\partial U_{r}}{\partial n}\right] n_{r}+\frac{\partial U_{r}}{\partial r} \\
\left(\frac{\partial \sigma_{r r}}{\partial \theta}\right)=\left[\frac{\partial \sigma_{r r}}{\partial n}\right] n_{\theta}+\frac{\partial \sigma_{r r}}{\partial \theta} \\
\left(\frac{\partial U_{r}}{\partial \theta}\right)=\left[\frac{\partial U_{r}}{\partial n}\right] n_{\theta}+\frac{\partial U_{r}}{\partial \theta} \\
\left(\frac{\partial \sigma_{\theta \theta}}{\partial r}\right)=\left[\frac{\partial \sigma_{\theta \theta}}{\partial n}\right] n_{r}+\frac{\partial \sigma_{\theta \theta}}{\partial r} \\
\left(\frac{\partial U_{\theta}}{\partial r}\right)=\left[\frac{\partial U_{\theta}}{\partial n}\right] n_{r}+\frac{\partial U_{\theta}}{\partial r} \\
\left(\frac{\partial \sigma_{\theta \theta}}{\partial \theta}\right)=\left[\frac{\partial \sigma_{\theta \theta}}{\partial n}\right] n_{\theta}+\frac{\partial \sigma_{\theta \theta}}{\partial \theta} \\
\left(\frac{\partial U_{\theta}}{\partial \theta}\right)=\left[\frac{\partial U_{\theta}}{\partial n}\right] n_{\theta}+\frac{\partial U_{\theta}}{\partial \theta} \\
\left(\frac{\partial \sigma_{r \theta}}{\partial \theta}\right)=\left[\frac{\partial \sigma_{r \theta}}{\partial n}\right] n_{\theta}+\frac{\partial \sigma_{r \theta}}{\partial \theta}
\end{array}\right\}
$$

where, in the case of cylindrical coordinate system, the components of the unit vector normal to the wave surface $\Phi$ are $n_{r}$ and $n_{\theta}$, which are defined to be

$$
\begin{aligned}
& n_{r}=\frac{\frac{\partial \Phi}{\partial r}}{\sqrt{\left(\frac{\partial \Phi}{\partial r}\right)^{2}+\frac{1}{r^{2}}\left(\frac{\partial \Phi}{\partial \theta}\right)^{2}}} \\
& n_{\theta}=\frac{\frac{\partial \Phi}{\partial \theta}}{\sqrt{\left(\frac{\partial \Phi}{\partial r}\right)^{2}+\frac{1}{r^{2}}\left(\frac{\partial \Phi}{\partial \theta}\right)^{2}}}
\end{aligned}
$$


such that

$$
n_{r}^{2}+\frac{n_{\theta}^{2}}{r^{2}}=1
$$

and let

$$
\left.\begin{array}{l}
n_{r}=\cos \theta \\
\frac{n_{\theta}}{r}=\sin \theta
\end{array}\right\}
$$

Analogous to Eqs. (46) and in view of Eqs. (78) through (88), the following system of five differential equations with the five unknowns

$$
\left[\frac{\partial U_{r}}{\partial n}\right],\left[\frac{\partial U_{\theta}}{\partial n}\right],\left[\frac{\partial \sigma_{r r}}{\partial n}\right],\left[\frac{\partial \sigma_{\theta \theta}}{\partial n}\right] \text {, and }\left[\frac{\partial \sigma_{r \theta}}{\partial n}\right]
$$

is obtained:

$$
\begin{gathered}
n_{r}\left[\frac{\partial \sigma_{r r}}{\partial n}\right]+\frac{n_{\theta}}{r}\left[\frac{\partial \sigma_{r \theta}}{\partial n}\right]+\rho G\left[\frac{\partial U_{r}}{\partial n}\right] \\
=-\frac{\sigma_{r r}-\sigma_{\theta \theta}}{r}+R_{U_{r}} \\
\frac{n_{\theta}}{r}\left[\frac{\partial \sigma_{\theta \theta}}{\partial n}\right]+n_{r}\left[\frac{\partial \sigma_{r \theta}}{\partial n}\right]+\rho G\left[\frac{\partial U}{\partial n}\right] \\
=-\frac{2 \sigma_{r \theta}}{r}+R_{U_{\theta}} \\
G\left[\frac{\partial \sigma_{r r}}{\partial n}\right]+(\lambda+2 \mu) n_{r}\left[\frac{\partial U_{r}}{\partial n}\right]+\frac{\lambda n_{\theta}}{r}\left[\frac{\partial U_{\theta}}{\partial n}\right] \\
=-\frac{\lambda U_{r}}{r}+R_{r r} \\
G\left[\frac{\partial \sigma_{\theta \theta}}{\partial n}\right]+\lambda n_{r}\left[\frac{\partial U_{r}}{\partial n}\right]+(\lambda+2 \mu) \frac{n_{\theta}}{r}\left[\frac{\partial U_{\theta}}{\partial n}\right] \\
=-(\lambda+2 \mu) \frac{U_{r}}{r}+R_{\theta \theta} \\
G\left[\frac{\partial \sigma_{r \theta}}{\partial n}\right]+\frac{\mu n_{\theta}}{r}\left[\frac{\partial U_{r}}{\partial n}\right]+\mu n_{r}\left[\frac{\partial U_{\theta}}{\partial n}\right] \\
=\frac{\mu U_{\theta}}{r}+R_{r \theta}
\end{gathered}
$$

where

$$
\begin{aligned}
\boldsymbol{R}_{U_{r}}= & \rho \frac{d U_{r}}{d t}-\rho G\left(\frac{\partial U_{r}}{\partial r} n_{r}+\frac{1}{r^{2}} \frac{\partial U_{r}}{\partial \theta} n_{\theta}\right) \\
& -\frac{\partial \sigma_{r r}}{\partial r}-\frac{1}{r} \frac{\partial \sigma_{r \theta}}{\partial \theta}
\end{aligned}
$$

$$
\begin{aligned}
R_{U_{\theta}}= & \rho \frac{d U_{\theta}}{d t}-{ }_{\rho} G\left(\frac{\partial U_{\theta}}{\partial r} n_{r}+\frac{1}{r^{2}} \frac{\partial U_{\theta}}{\partial \theta} n_{\theta}\right) \\
& -\frac{1}{r} \frac{\partial \sigma_{\theta \theta}}{\partial \theta}-\frac{\partial \sigma_{r \theta}}{\partial r} \\
R_{r r}= & \frac{d \sigma_{r r}}{d t}-G\left(\frac{\partial \sigma_{r r}}{\partial r} n_{r}+\frac{1}{r^{2}} \frac{\partial \sigma_{r r}}{\partial \theta} n_{\theta}\right) \\
& -(\lambda+2 \mu) \frac{\partial U_{r}}{\partial r}-\frac{\lambda}{r} \frac{\partial U_{\theta}}{\partial \theta} \\
R_{\theta \theta}= & \frac{d \sigma_{\theta \theta}}{d t}-G\left(\frac{\partial \sigma_{\theta \theta}}{\partial r} n_{r}+\frac{1}{r^{2}} \frac{\partial \sigma_{\theta \theta}}{\partial \theta} n_{\theta}\right) \\
& -(\lambda+2 \mu) \frac{1}{r} \frac{\partial U_{\theta}}{\partial \theta}-\lambda \frac{\partial U_{r}}{\partial r} \\
R_{r \theta}= & \frac{d \sigma_{r \theta}}{d t}-G\left(\frac{\partial \sigma_{r \theta}}{\partial r} n_{r}+\frac{1}{r^{2}} \frac{\partial \sigma_{r \theta}}{\partial \theta} n_{\theta}\right) \\
& -\mu\left(\frac{\partial U_{\theta}}{\partial r}+\frac{1}{r} \frac{\partial U_{r}}{\partial \theta}\right)
\end{aligned}
$$

and where

$$
G=n_{r} \frac{d r}{d t}+n_{\theta} \frac{d \theta}{d t}
$$

By the same steps as for the Cartesian coordinate system, the following five characteristics are obtained:

$$
\begin{aligned}
& G_{1}=\sqrt{\frac{\lambda+2 \mu}{\rho}} \\
& G_{2}=-\sqrt{\frac{\lambda+2 \mu}{\rho}} \\
& G_{3}=\sqrt{\frac{\mu}{\rho}} \\
& G_{4}=-\sqrt{\frac{\mu}{\rho}} \\
& G_{5}=0
\end{aligned}
$$


which are identical to Eqs. (50) through (54). The bicharacteristic curves may be found simply by transferring Eq. (57) into cylindrical coordinates, as follows:

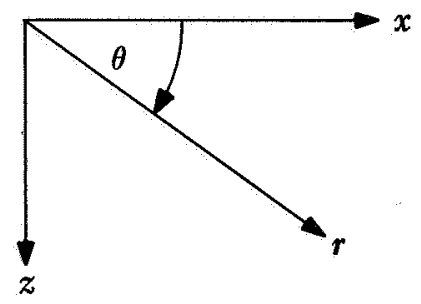

where

$$
\begin{aligned}
& x=r \cos \theta \\
& z=r \sin \theta
\end{aligned}
$$

thus,

$$
\begin{aligned}
& d x=\cos \theta d r-r \sin \theta d \theta \\
& d z=\sin \theta d r+r \cos \theta d \theta
\end{aligned}
$$

Equation (57) now becomes

$$
(d r)^{2}+r^{2}(d \theta)^{2}=G^{2}(d t)^{2}
$$

and the bicharacteristic curves are

$$
\begin{aligned}
d r & =G \cos \theta d t \\
r d \theta & =G \sin \theta d t
\end{aligned}
$$

or

$$
\begin{aligned}
& d r=n_{r} G d t \\
& d \theta=\frac{n_{\theta}}{r^{2}} G d t
\end{aligned}
$$

Now, following steps similar to those described in Section VI, one obtains the desired five characteristic equations along the bicharacteristics:

$$
\begin{aligned}
n_{r}^{2} R_{r r}+ & \frac{n_{\theta}^{2}}{r^{2}} R_{\theta \theta}+2 \frac{n_{r} n_{\theta}}{r} R_{r \theta}-G_{L} n_{r} R_{U_{r}}-G_{L} \frac{n_{\theta}}{r} R_{U_{\theta}} \\
= & \frac{\left(n_{r} d r+n_{\theta} d \theta\right)}{r d t}\left[\left(\sigma_{\theta \theta}-\sigma_{r r}\right) n_{r}-2 \sigma_{r_{\theta}} \frac{n_{\theta}}{r}\right] \\
& +\frac{1}{r}\left[\left(\lambda+2 \mu \frac{n_{\theta}^{2}}{r^{2}}\right) U_{r}-\frac{2 n_{r} n_{\theta}}{r} \mu U_{\theta}\right]
\end{aligned}
$$

along

$$
\begin{gathered}
\frac{d r}{d t}=G_{L} n_{r} \\
\frac{d \theta}{d t}=G_{L} \frac{n_{\theta}}{r^{2}} \\
n_{r}^{2} R_{r r}+\frac{n_{\theta}^{2}}{r^{2}} R_{\theta \theta}+2 \frac{n_{r} n_{\theta}}{r} R_{r \theta}+G_{L} n_{r} R_{U_{r}}+G_{L} \frac{n_{\theta}}{r} R_{U_{\theta}} \\
=\frac{\left(n_{r} d r+n_{\theta} d \theta\right)}{r d t}\left[\left(\sigma_{\theta \theta}-\sigma_{r r}\right) n_{r}-2 \sigma_{r \theta} \frac{n_{\theta}}{r}\right] \\
+\frac{1}{r}\left[\left(\lambda+2 \mu \frac{n_{\theta}^{2}}{r^{2}}\right) U_{r}-\frac{2 n_{r} n_{\theta}}{r} \mu U_{\theta}\right]
\end{gathered}
$$

along

$$
\begin{gathered}
\frac{d r}{d t}=-G_{L} n_{r} \\
\frac{d \theta}{d t}=-G_{L} \frac{n_{\theta}}{r^{2}} \\
\frac{n_{\theta} n_{r}}{r} R_{r r}-\frac{n_{\theta} n_{r}}{r} R_{\theta \theta}-\left(n_{r}^{2}-\frac{n_{\theta}^{2}}{r^{2}}\right) R_{r \theta}-G_{S} \frac{n_{\theta}}{r} R_{V_{r}} \\
+G_{S} n_{r} R_{U_{\theta}}=\frac{\left(n_{r} d r+n_{\theta} d \theta\right)}{r d t}\left[\left(\sigma_{\theta \theta}-\sigma_{r r}\right) \frac{n_{\theta}}{r}+2 \sigma_{r \theta} n_{r}\right. \\
\left.-2 U_{r \rho} G_{S} \frac{n_{r} n_{\theta}}{r}+U_{\theta \rho} G_{r S}\left(n_{r}^{2}-\frac{n_{\theta}^{2}}{r^{2}}\right)\right]
\end{gathered}
$$

along

$$
\begin{gathered}
\frac{d r}{d t}=G_{S} n_{r} \\
\frac{d \theta}{d t}=G_{S} \frac{n_{\theta}}{r^{2}} \\
\frac{n_{\theta} n_{r}}{r} R_{r r}-\frac{n_{\theta} n_{r}}{r} R_{\theta \theta}-\left(n_{r}^{2}-\frac{n_{\theta}^{2}}{r^{2}}\right) R_{r \theta}+G_{S} \frac{n_{\theta}}{r} R_{U_{r}} \\
-G_{S} n_{r} R_{U_{\theta}}=\frac{\left(n_{r} d r+n_{\theta} d \theta\right)}{r d t}\left[\left(\sigma_{\theta \theta}-\sigma_{r r}\right) \frac{n_{\theta}}{r}+2 \sigma_{r \theta} n_{r}\right. \\
\left.+2 U_{r \rho} G_{s} \frac{n_{r} n_{\theta}}{r}-U_{\theta \rho} G_{S}\left(n_{r}^{2}-\frac{n_{\theta}^{2}}{r^{2}}\right)\right]
\end{gathered}
$$


along

$$
\begin{gathered}
\frac{d r}{d t}=-G_{\mathrm{S}} n_{r} \\
\frac{d \theta}{d t}=-G_{\mathrm{S}} \frac{n_{\theta}}{r^{2}} \\
{\left[\lambda n_{r}^{2}-\frac{n_{\theta}^{2}}{r^{2}}(\lambda+2 \mu)\right] R_{r r}+\left[\lambda \frac{n_{\theta}^{2}}{r^{2}}-n_{r}^{2}(\lambda+2 \mu)\right] \boldsymbol{R}_{\theta \theta}} \\
+4 \frac{n_{r} n_{\theta}}{r}(\lambda+\mu) R_{r \theta} \\
=\frac{1}{r}\left\{U_{r}\left[\frac{n_{\theta}^{2}}{r^{2}} \lambda(\lambda+2 \mu)-4 \mu n_{r}^{2}(\lambda+\mu)\right]\right. \\
\left.+4 \frac{U_{\theta}}{r} n_{r} n_{\theta} \mu(\lambda+\mu)\right\}
\end{gathered}
$$

along

$$
\begin{aligned}
& d r=0 \\
& d \theta=0
\end{aligned}
$$

where

$$
\left.\begin{array}{l}
G_{L}=\sqrt{\frac{\lambda+2 \mu}{\rho}} \\
G_{S}=\sqrt{\frac{\mu}{\rho}}
\end{array}\right\}
$$

and where the $R$ are expressed in Eqs. (94) when the relevant $G$ is inserted in these equations.

With the availability of the derived Cartesian characteristic equations (68) through (72) and the cylindrical characteristic equations (104) through (109), two-spatial dimensional wave-propagation problems can be analyzed. Such problems are discussed in Section VIII, and two problems are solved in Section IX.

\section{Indicated Applications of the Derived Characteristic Equations}

The purpose of this section is to indicate the applications of the derived characteristic equations to a wide range of boundary-value problems. First, as a measure of validity, the derived two-spatial dimensional characteristic equations are shown upon reduction to contain the known one-spatial dimensional form. It is then shown that the derived two-spatial dimensional characteristic equations accommodate more complex cases encountered in one-spatial dimensional wave problems. Finally, twospatial dimensional problems which lend themselves to the derived equations are indicated.

\section{A. A Known One-Spatial Dimensional Problem}

Before the applications of the derived characteristic equations are discussed, a known one-spatial dimensional problem is considered in order to measure the validity of these equations. The problem consists of a circular cylindrical cavity in an infinite elastic solid subjected to a uniform radial load applied to the cavity wall (Fig. 7).

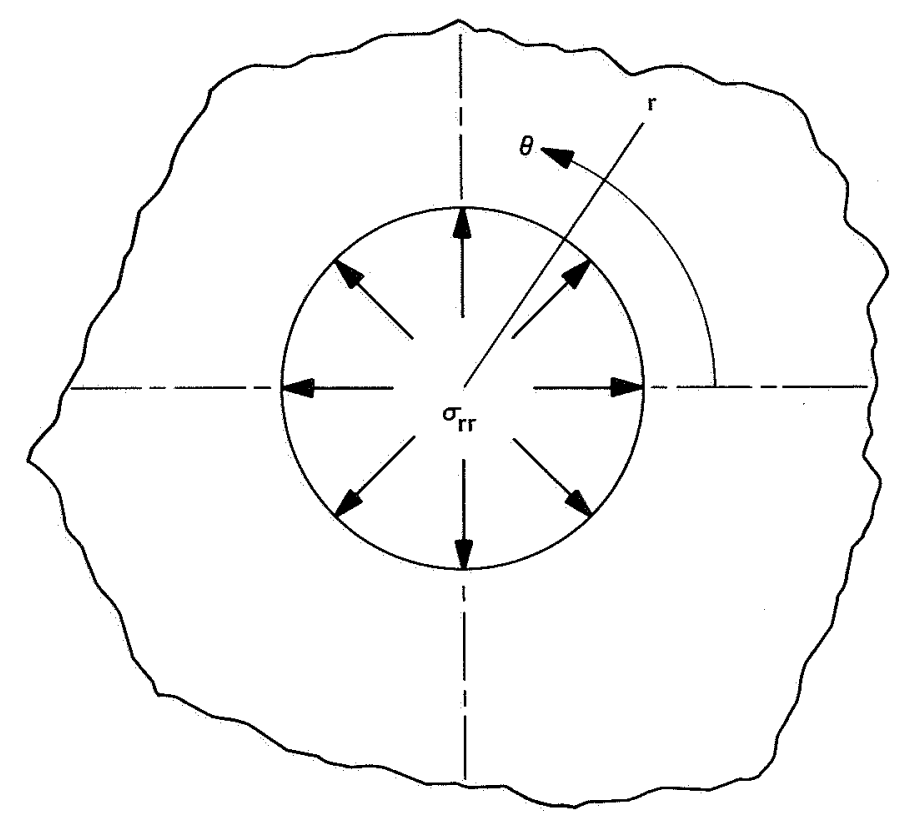

Fig. 7. Circular cylindrical cavify in an infinite elastic solid subjected to a uniform radial load applied to cavity wall

It is readily seen that the unknown dependent variables involved here are $\sigma_{r r}, \sigma_{\theta \theta}$, and $U_{r}\left(\sigma_{r \theta}=U_{\theta}=0\right)$, which are the functions of $r$ only. Thus, from Eqs. (89), (90), and (91), it is seen that $n_{\theta}=0$ and $n_{r}=1$. Also, from Eq. (59), when $x$ and $z$ are replaced by $r$ and $\theta$, it is seen that the only possible $G$ are $G_{1}^{ \pm}= \pm[(\lambda+2 \mu) / \rho]^{1 / 2}$. It follows from Eqs. (104) through (109) that the characteristic equations for this problem are

$$
d \sigma_{r r}-{ }_{\rho} G_{\bar{L}}^{ \pm} d U_{r}=\left(\sigma_{\theta \theta}-\sigma_{r r}+{ }_{\rho} G_{\bar{L}}^{ \pm} \frac{\lambda}{\lambda+2 \mu} U_{r}\right) \frac{d r}{r}
$$


along $d r / d t=G_{L}$, where

$$
\begin{gathered}
G_{t}^{ \pm}= \pm \sqrt{\frac{\lambda+2 \mu}{\rho}} \\
\frac{d \sigma_{r r}}{d \sigma_{\theta \theta}}=\frac{\lambda+2 \mu}{\lambda}\left[1-\frac{4 \mu(\lambda+\mu)}{\lambda+2 \mu} \frac{U_{r}}{r} \frac{d t}{d \sigma_{\theta \theta}}\right]
\end{gathered}
$$

along $d r=0$, which are indeed Eqs. (18) and (19) (p. 161) in Chou and Koenig's paper (Ref. 4). Also, it can be shown that Eqs. (68) through (72) contain, upon reduction, known one-spatial dimensional cases that require Cartesian characteristic equations.

\section{B. A Uniform Plane Oblique Load Applied on a Half-Space}

This problem consists of an elastic half-space subjected to a uniform plane oblique load (Fig. 8). Although the problem is a one-spatial dimensional one, it may be considered a step toward a strictly two-spatial dimensional problem in the sense of having two kinds of waves and five unknown dependent variables. The unknown dependent variables are $\sigma_{x x}, \sigma_{z z}, \sigma_{x z}, U$, and $W$, which are functions of $z$ only. It can readily be seen that a shear wave and a longitudinal wave will be generated here. In view of these observations, the characteristic equations (68) through (72) will immediately be reduced to the following:

$$
d \sigma_{z z}-\rho \sqrt{\frac{\lambda+2 \mu}{\rho}} d W=0
$$

along

$$
\begin{gathered}
\frac{d z}{d t}=\sqrt{\frac{\lambda+2 \mu}{\rho}} \\
d \sigma_{z z}+\rho \sqrt{\frac{\lambda+2 \mu}{\rho}} d W=0
\end{gathered}
$$

along

$$
\begin{gathered}
\frac{d z}{d t}=-\sqrt{\frac{\lambda+2 \mu}{\rho}} \\
d \sigma_{x z}-\rho \sqrt{\frac{\mu}{\rho}} d U=0
\end{gathered}
$$

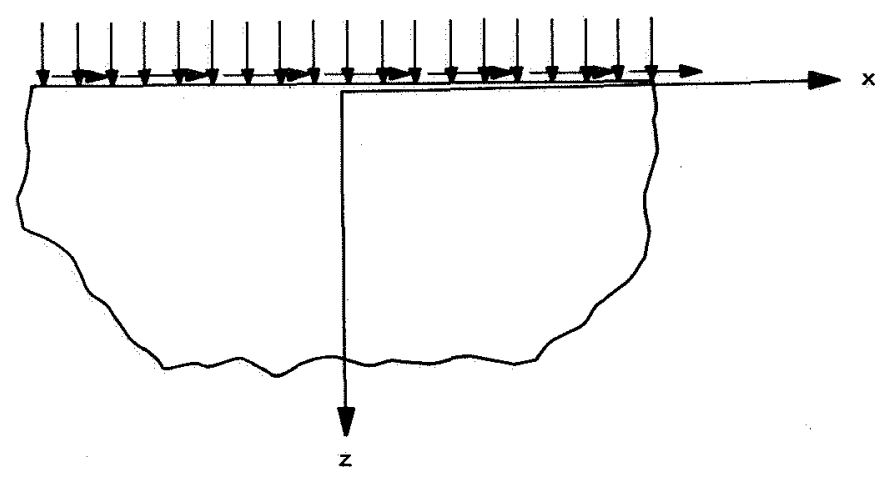

Fig. 8. Uniform plane oblique load applied on a half-space

along

$$
\begin{gathered}
\frac{d z}{d t}=\sqrt{\frac{\mu}{\rho}} \\
d \sigma_{x z}+\rho \sqrt{\frac{\mu}{\rho}} d U=0
\end{gathered}
$$

along

$$
\frac{d z}{d t}=-\sqrt{\frac{\mu}{\rho}}
$$

and

$$
\sigma_{x x}=\frac{\lambda}{\lambda+2 \mu} \sigma_{z z}
$$

and now the numerical evaluations are possible.

\section{A Circular Cylindrical Cavity Subjected to a Uniform Radial Load and a Uniform Tangential Load}

This problem consists of a circular cylindrical cavity, in an infinite elastic solid, subjected simultaneously to a uniform radial load and a uniform tangential load applied to the cavity wall (Fig. 9). Like the preceding example, this is also a one-spatial dimensional problem. It can be seen that the five unknown dependent variables $\sigma_{r r}, \sigma_{\theta \theta}$, $\sigma_{r \theta}, U_{r}$, and $U_{\theta}$ involved are functions of $r$ only. Two kinds of waves exist here also, namely, a shear wave and a longitudinal wave. In view of these observations, Eqs. (104) through (109) are reduced to the following characteristic equations:

$$
d \sigma_{r r}-\rho G_{L} d U_{r}=\left(\sigma_{\theta \theta}-\sigma_{r r}+\rho G_{L} \frac{\lambda}{\lambda+2 \mu} U_{r}\right) \frac{d r}{r}
$$




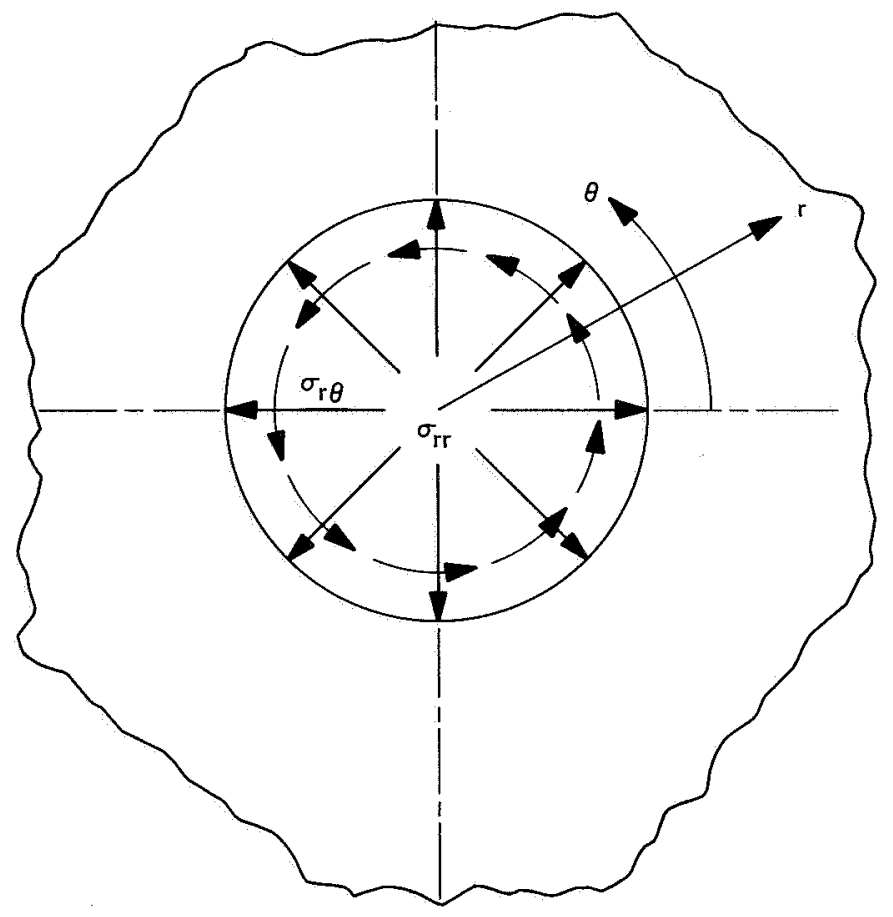

Fig. 9. Circular cylindrical cavity subjected to a uniform radial load and a uniform fangential load

along

$$
\begin{gathered}
\frac{d r}{d t}=G_{L} \\
d \sigma_{r r}+{ }_{\rho} G_{L} d U_{r}=\left(\sigma_{\theta \theta}-\sigma_{r r}-\rho G_{L} \frac{\lambda}{\lambda+2 \mu} U_{r}\right) \frac{d r}{r}
\end{gathered}
$$

along

$$
\begin{aligned}
& \frac{d r}{d t}=-G_{L} \\
& d \sigma_{r \theta}-\rho G_{s} d U_{\theta}=-\left(2 \sigma_{r \theta}+\rho G_{s} U_{\theta}\right) \frac{d r}{r}
\end{aligned}
$$

along

$$
\begin{gathered}
\frac{d r}{d t}=G_{S} \\
d \sigma_{r \theta}+\rho G_{S} d U_{\theta}=-\left(2 \sigma_{r \theta}-\rho G_{S} U_{\theta}\right) \frac{d r}{r}
\end{gathered}
$$

along

$$
\begin{gathered}
\frac{d r}{d t}=-G_{S} \\
\frac{d \sigma_{r r}}{d \sigma_{\theta \theta}}=\frac{\lambda+2 \mu}{\lambda}\left[1-\frac{4 \mu(\lambda+\mu)}{\lambda+2 \mu} \frac{U_{r}}{r} \frac{d t}{d \sigma_{\theta \theta}}\right]
\end{gathered}
$$

along

$$
d r=0
$$

where

$$
\left.\begin{array}{l}
G_{L}=\sqrt{\frac{\lambda+2 \mu}{\rho}} \\
G_{S}=\sqrt{\frac{\mu}{\rho}}
\end{array}\right\}
$$

With Eqs. (117) through (122), the numerical evaluation is now possible.

\section{A Line Load Applied on a Half-Space}

This problem also contains five dependent variables and two kinds of waves (see Fig. 15). However, the dependent variables here are functions of two-spatial dimensions. Equations (68) through (72) include all their terms. H. Lamb (Ref. 12) was the first to deal with the problem, which, in the past, has been treated by the transform techniques. A summary of work by other investigators who use the transform techniques is presented in Ref. 1 (pp. 214-231). In Section IX of this report, the problem is treated by the theory of characteristics.

\section{E. A Circular Cylindrical Cavity Subjected to a Line Load}

Miklowitz (Ref. 13) solved this problem by the transform techniques. When treated by the theory of characteristics, the problem seems to lend itself to the cylindrical characteristics equations (104) through (109).

\section{Solutions to Specific Boundary-Value Problems}

Based on the problems suggested in Section VII, two boundary-value problems are solved here. The derived characteristic equations are applied to the numerical evaluation of these two representative problems.

The first problem consists of an infinite elastic flat plate impacted by a uniform plane oblique load. The second problem deals with an elastic half-space impacted by a line load. In both cases, the load is applied abruptly to the solids. Consequently, discontinuities may occur in the dependent variables themselves across the leading wave front (the first generated wave surface) (Ref. 4). The characteristic equations, however, were derived for conditions under which discontinuities are allowed only in the first partial derivatives of the dependent variables. Therefore, the characteristic equations are reworked here 
to accommodate the leading wave front. Once the dependent variables are computed along the leading wave front, the rest of the solution domain can be evaluated numerically by direct employment of the appropriate derived characteristic equations (Eqs. 68 through 72 or 104 through 108). In cases in which the load is applied gradually, rather than abruptly, the characteristic equations can be applied directly to the entire solution domain.

\section{A. A Uniform Plane Oblique Load Suddenly Applied on an Infinite Flat Plate}

A detailed solution to a specific problem, similar to the outlined example discussed in Section VIII, is solved here.

A bounded half-space is impacted by step normal and tangential velocities, as described in Figs. 10 and 11 and Eq. (123). This combined step load is uniformly distributed over the surface $z=0$.

$$
\bar{U}, \bar{W}=\left\{\begin{array}{l}
1 \text { when } \tau \supseteq 0 \\
0 \text { when } \tau<0
\end{array}\right.
$$

where $\bar{U}, \bar{W}$, and $\tau$ are dimensionless quantities:

$$
\begin{gathered}
\bar{U}=\frac{U}{G_{L}} \\
\bar{W}=\frac{W}{G_{L}} \\
\tau=\frac{G_{L} t}{L}
\end{gathered}
$$

and

$$
\begin{aligned}
\bar{x} & =\frac{x}{L} \\
\bar{z} & =\frac{z}{L} \\
\overline{\boldsymbol{\sigma}}_{x x} & =\frac{\sigma_{x x}}{\lambda+2 \mu} \\
\overline{\boldsymbol{\sigma}}_{z x} & =\frac{\sigma_{z z}}{\lambda+2 \mu} \\
\overline{\boldsymbol{\sigma}}_{z z} & =\frac{\sigma_{z z}}{\lambda+2 \mu} \\
\bar{\sigma}_{y y} & =\frac{\sigma_{y y}}{\lambda+2 \mu}
\end{aligned}
$$

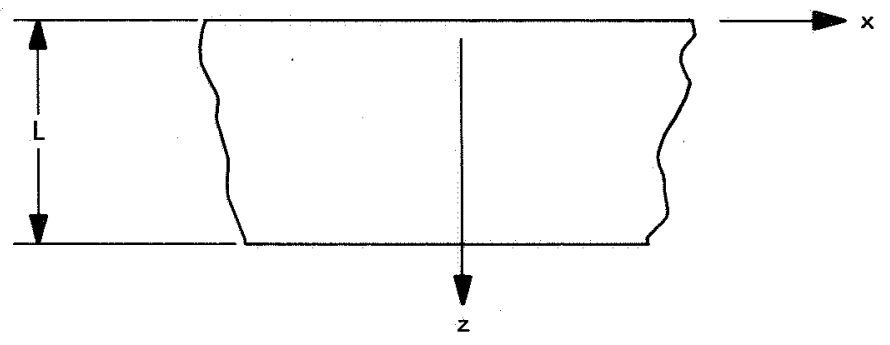

Fig. 10. Bounded half-space

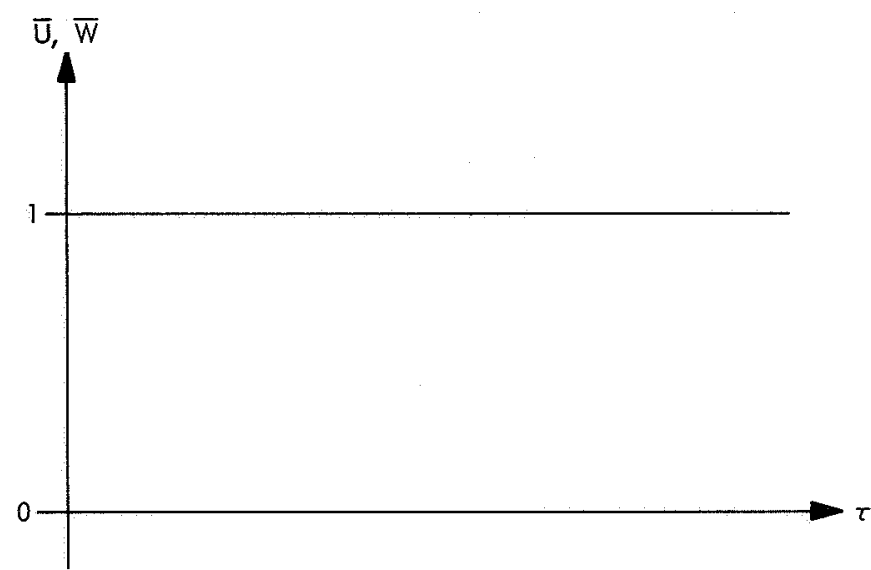

Fig. 11. Step normal and tangential velocities

This problem may simulate, for example, an impact landing of an infinite flat plate on a rigid target at rest (Fig. 12a)'. For the convenience of having zero initial conditions, an oblique velocity $\mathbf{V}$, such that $\mathbf{V}=\mathbf{W}+\mathbf{U}$, is added to the whole system (Fig. 12b). This implies that the motion is relative to the initial steady motion of the plate.

It follows that at $\tau=0$, the rigid target is suddenly impacting on the plate, and in this instant $(r=0)$, the plate and the target are at rest. Since the given actual velocity of the plate is constant, the added constant velocity will not affect the emanating stress waves.

The following analysis is done by using the Lagrangian viewpoint; i.e., an observer is assigned to the origin of the coordinate system at the impact face of the plate $(z=0)$. The observer fixes his attention on a specific particle in the plate at time zero, when the plate is still at rest and therefore unstrained. This implies that the stresses will be measured from the initial unstrained condition. 


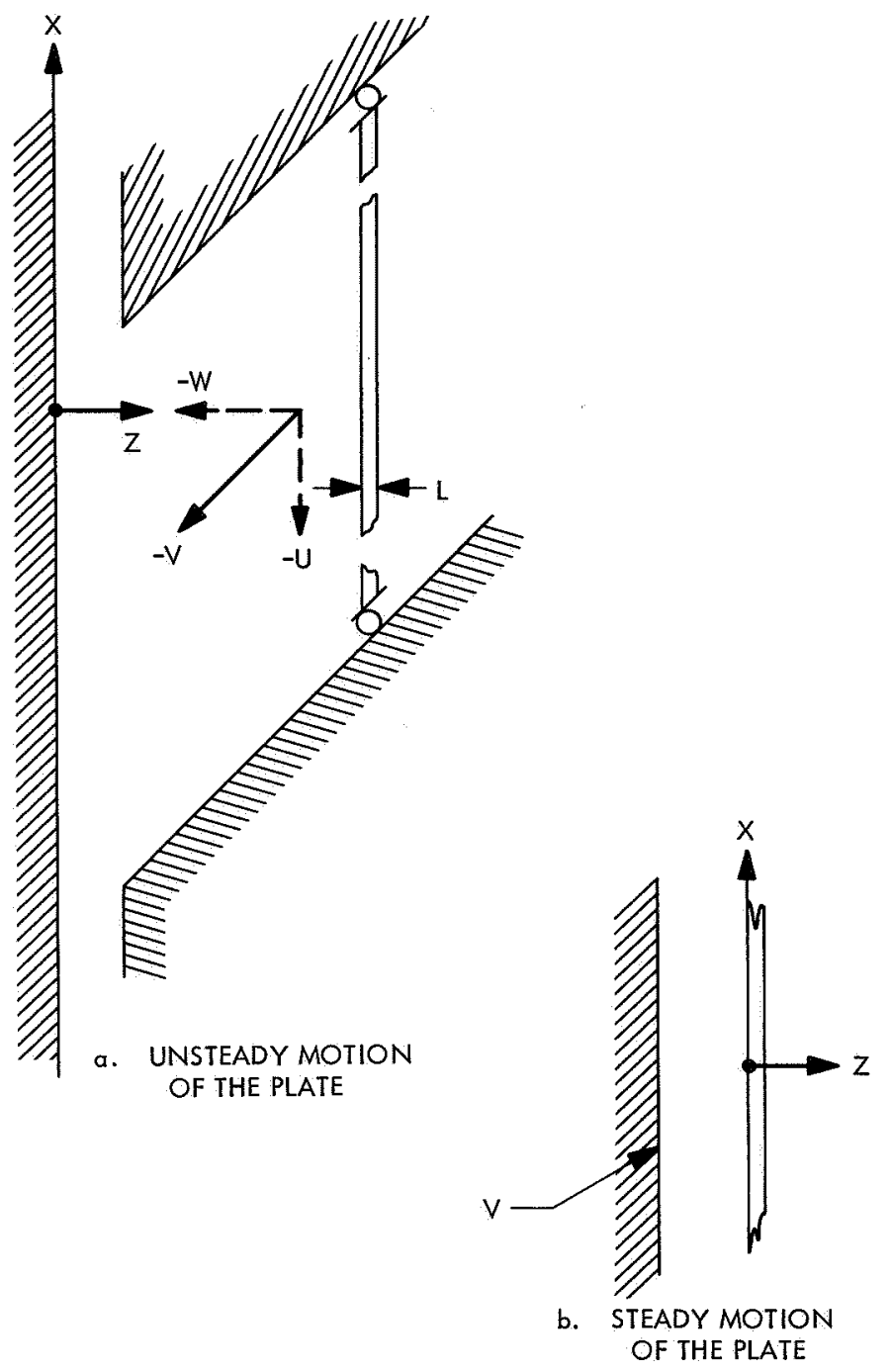

Fig. 12. Infinite flat plate landing on a rigid target

The initial conditions are then

$$
\left.\begin{array}{rl}
\bar{V} & =0 \\
\bar{\sigma}_{i j} & =0
\end{array}\right\} \text { at } \tau=0 \text { and } 0 \leq \bar{z} \leq 1
$$

and the boundary conditions are

$$
\left.\begin{array}{rl}
\bar{V} & =\sqrt{2} \text { at } \bar{z}=0 \\
\bar{\sigma}_{i j} & =0 \quad \text { at } \bar{z}=1
\end{array}\right\} \text { for } \tau>0
$$

In accordance with the discussion in Section VIII and Eqs. (112) through (116), the following four ordinary differential equations which relate the four unknown func- tions $\left(\overline{\boldsymbol{\sigma}}_{z z}, \overline{\boldsymbol{\sigma}}_{z x}, \overline{\mathrm{U}}, \bar{W}\right)$ along the bicharacteristic curves are (in dimensionless form):

$$
\begin{array}{rc}
d \bar{\sigma}_{z z}-d \bar{W}=0 & \text { along } \frac{\overline{d z}}{d \tau}=1 \\
d \bar{\sigma}_{z z}+d \bar{W}=0 & \text { along } \frac{\overline{d z}}{d \tau}=-1 \\
d \bar{\sigma}_{z x}-\frac{G_{s}}{G_{L}} d \bar{U}=0 & \text { along } \frac{\overline{d z}}{d \tau}=\frac{G_{S}}{G_{L}} \\
d \bar{\sigma}_{z x}+\frac{G_{S}}{G_{L}} d \bar{U}=0 & \text { along } \frac{\overline{d z}}{d \tau}=-\frac{G_{S}}{G_{L}}
\end{array}
$$

also

$$
\overline{\boldsymbol{\sigma}}_{x x}=\overline{\boldsymbol{\sigma}}_{y y}=\frac{v}{1-v} \overline{\boldsymbol{\sigma}}_{z z}
$$

where $v$ is Poisson's ratio, which was chosen for this example to be equal to 0.25 . Hence, Eqs. (127) through (131) become, after bars have been dropped,

$$
\begin{array}{cc}
d \sigma_{z z}-d W=0 & \text { along } \frac{d z}{d \tau}=1 \\
d \sigma_{z z}+d W=0 & \text { along } \frac{d z}{d \tau}=-1 \\
d \sigma_{z x}-\frac{1}{\sqrt{3}} d U=0 & \text { along } \frac{d z}{d \tau}=\frac{1}{\sqrt{3}} \\
d \sigma_{z x}+\frac{1}{\sqrt{3}} d U=0 & \text { along } \frac{d z}{d \tau}=-\frac{1}{\sqrt{3}} \\
\sigma_{x z}=\sigma_{y y}=\frac{1}{3} \sigma_{z z} \\
\sigma_{x y}=\sigma_{z y}=0
\end{array}
$$

To evaluate the dependent variables in the solution domain numerically, it is first necessary to compute them along the leading wave front. Because of the abrupt load, discontinuities may occur in the dependent variables along the leading wave front. Therefore, the characteristic equation (132), which holds along the first bicharacteristic curve $z=\tau$, has to be reformulated to accommodate the discontinuities in the variables themselves. Thus, in view 
of definition (27), Eq. (132), and Fig. 12, the following relation exists for point $P$ on the leading wave front:

$$
d\left[\sigma_{z z}\right]-d[W]=0
$$

Equation (138) is now integrated as the bicharacteristic curve $z-\tau=$ constant approaches the leading wave front $z-r=0$, i.e.,

$$
\left[\sigma_{z z}\right]-[W]=K
$$

where $K$ is the constant of integration. Since the material is at rest in front of the leading wave,

$$
\sigma_{z z_{\text {front }}}=W_{P_{\text {front }}}=0
$$

and Eq. (139) is rewritten as

$$
\sigma_{z z_{\text {rear }}}-W_{P_{\text {rear }}}=K
$$

On the other hand, the characteristic equation (133) 2. which holds along the bicharacteristic curve $z=-\tau+$ constant may be used as it stands for point $P$, since there are no abrupt changes across $z=-\tau+$ constant. Thus, the following relation exists:

$$
d \sigma_{z z}+d W=0 \quad \text { along } z=-\tau+\text { constant }
$$

or, by the finite difference method (Ref. 12),

$\left(\sigma_{z z_{\text {rear }}}-\sigma_{z z_{p_{\text {front }}}}\right)+\left(W_{P_{\text {rear }}}-W_{P_{\text {front }}}\right)=0$

Again, since $\sigma_{z z_{p_{\text {front }}}}=W_{P_{\text {front }}}=0$, relation (141) is written as

$$
\sigma_{z z_{\text {rear }}}=-W_{P_{\text {rear }}}
$$

In view of relations (140) and (142), it is concluded that $\sigma_{z z_{P_{\text {rear }}}}$ and $W_{\boldsymbol{P}_{\text {rear }}}$ remain constant along the leading wave front. Now, since the applied load at the origin $(z=0, \tau>0)$ is $W=1$, it follows from Eq. (142) that $\sigma_{z z}=-1$ at the origin. It follows immediately that $\sigma_{z z}$ and $W$ are equal to -1 and 1 , respectively, along the leading wave front $\left(\sigma_{z z_{P}}=-1\right.$ and $\left.W_{P}=1\right)$ until the wave front reaches the boundary $z=1$ (Fig. 13). The above analysis is then repeated for the reflected wave.

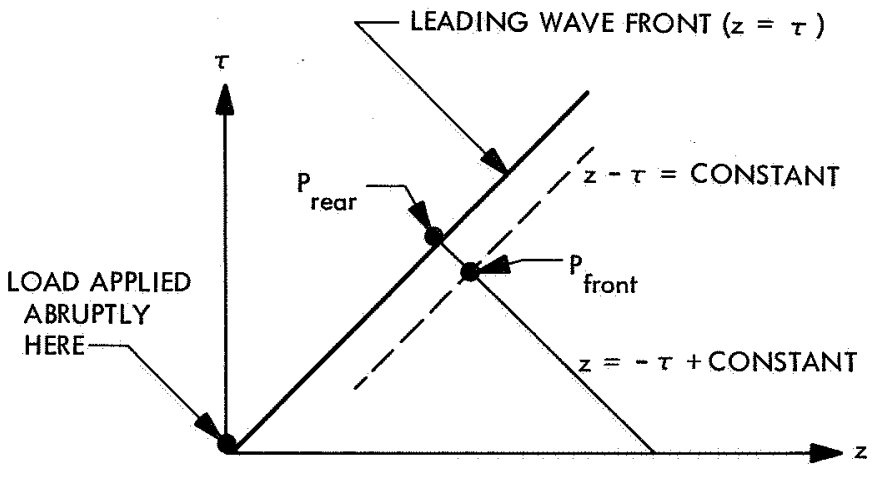

Fig. 13. Discontinuity in the dependent variables across the leading wave front

Equations (134) and (135) are treated similarly. Figures 14,15 , and 16 represent the stress and velocity distribution obtained in the $x z$-plane.

\section{B. A Line Load Suddenly Applied on a Half-Space}

The disturbance generated in a semi-infinite elastic medium by an impulsive load applied along an infinite line is considered here (See Refs. 1 and 12 for previous treatments of this problem by other methods.) The load is applied along the line $x=0, z=0$ in the $z$-direction (Figs. 17 and 18). The objective here is to determine the deformation which takes place on the surface of the halfspace at the leading wave front. All the variables involved are in the dimensionless form expressed in identities (124), and $v=0.25$. Hence, the only applied load is

$$
\left.\sigma_{z z}\right|_{\text {at } z=0}=1 \Delta(x) H(\tau)
$$

where

$$
\begin{aligned}
1 & =\text { concentrated load per unit length } \\
\Delta(x) & =\text { Dirac delta function (Ref. 1) } \\
H(\tau) & =\text { Heaviside's unit step function (see Fig. 17) }
\end{aligned}
$$

In order to evaluate the dependent variables for a material particle at a point $P(x, 0, \tau)$ on a free surface, a scheme is suggested which is depicted by Fig. 19. This scheme consists of rectangular $\left(\theta=0^{\circ}\right.$ and $\left.\theta=90^{\circ}\right)$ bicharacteristic curves (see Fig. 5) which initiate at points $A, B, D, E, F, M$, and $Q$ in the plane $\tau-\Delta \tau$ and terminate in the point $P(x, 0, \tau)$. The dependent variables of the material particles at points in the $\tau-\Delta \tau$ plane are known from initial prescribed conditions or from previous calculations. From these known points in the $\tau-\Delta \tau$ plane, $P(x, 0, \tau)$ generally is evaluated by the finite difference method (Ref. 14). 


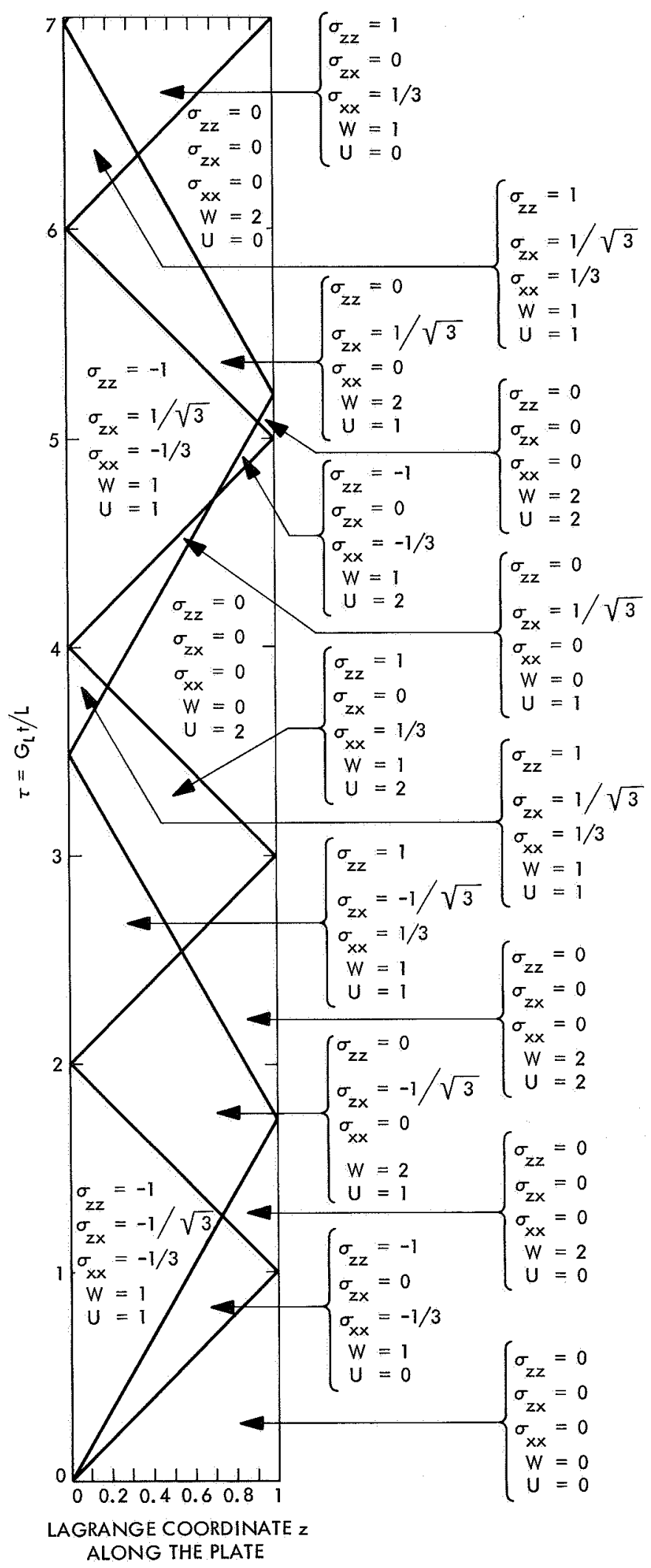

Fig. 14. Bicharacteristic grid 

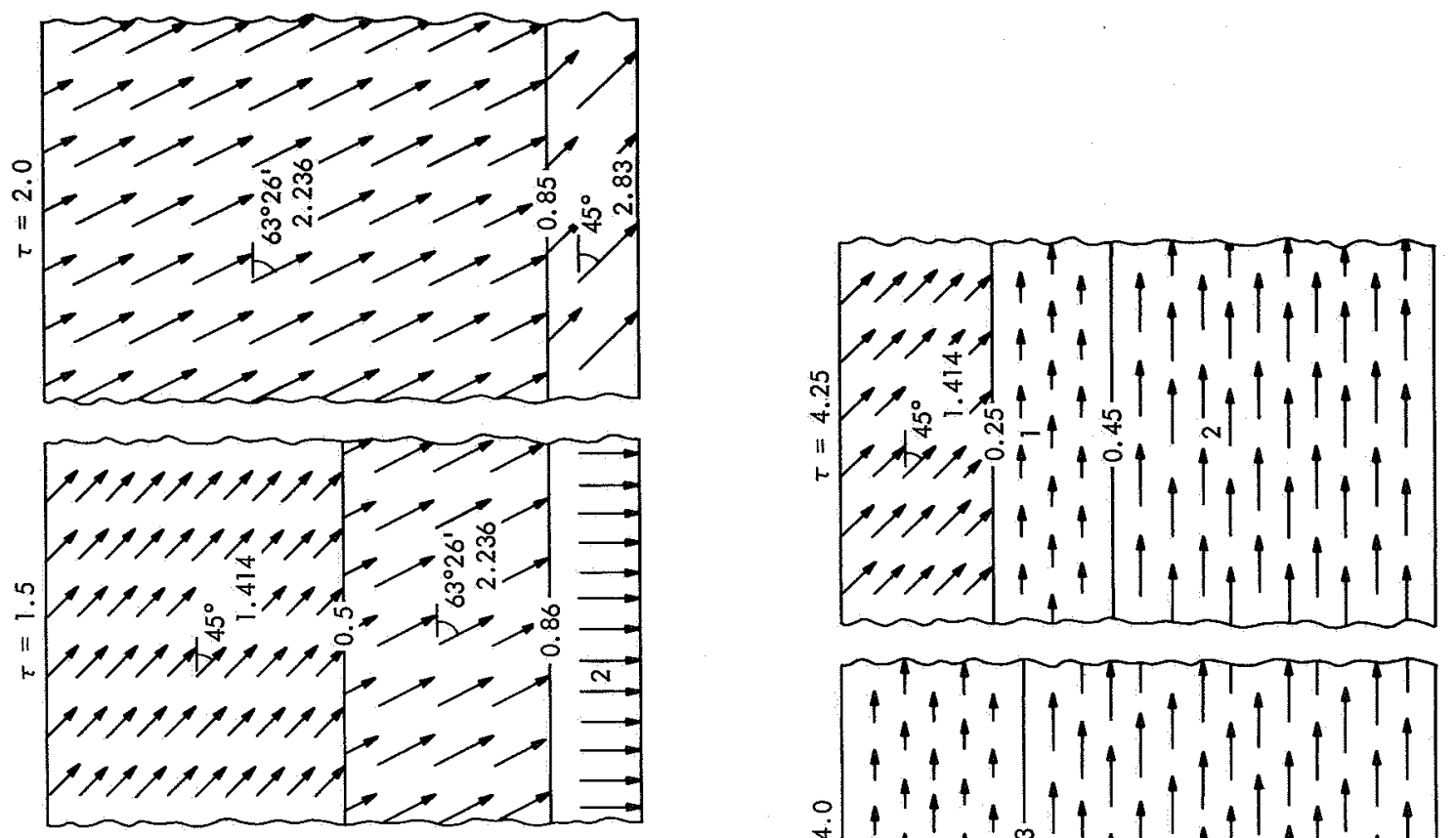

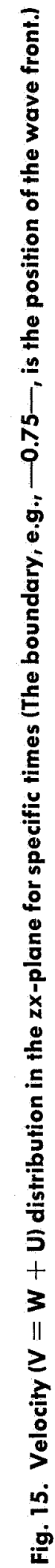
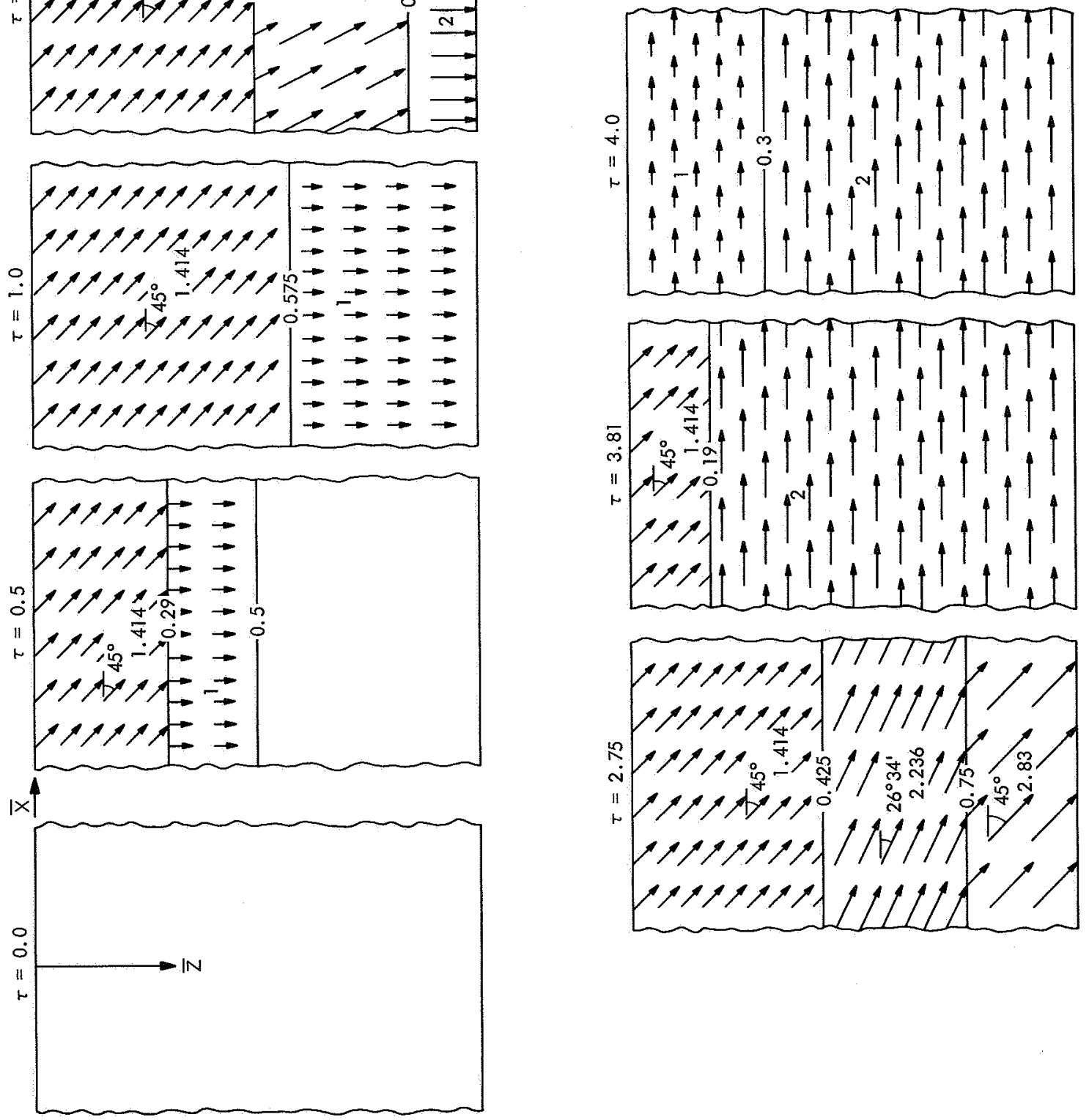

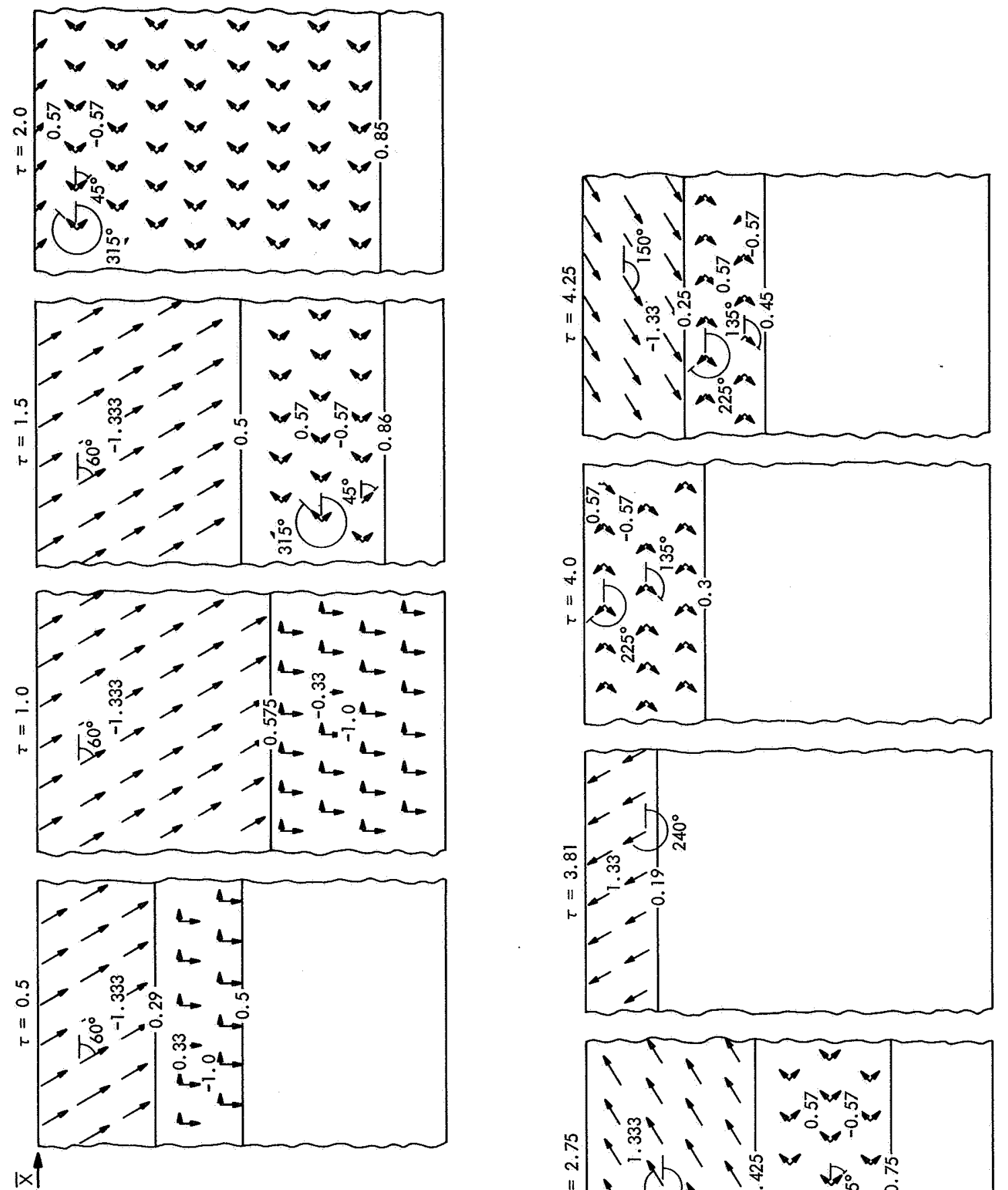

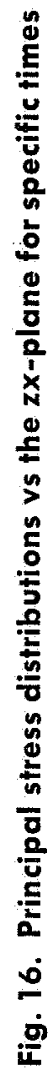
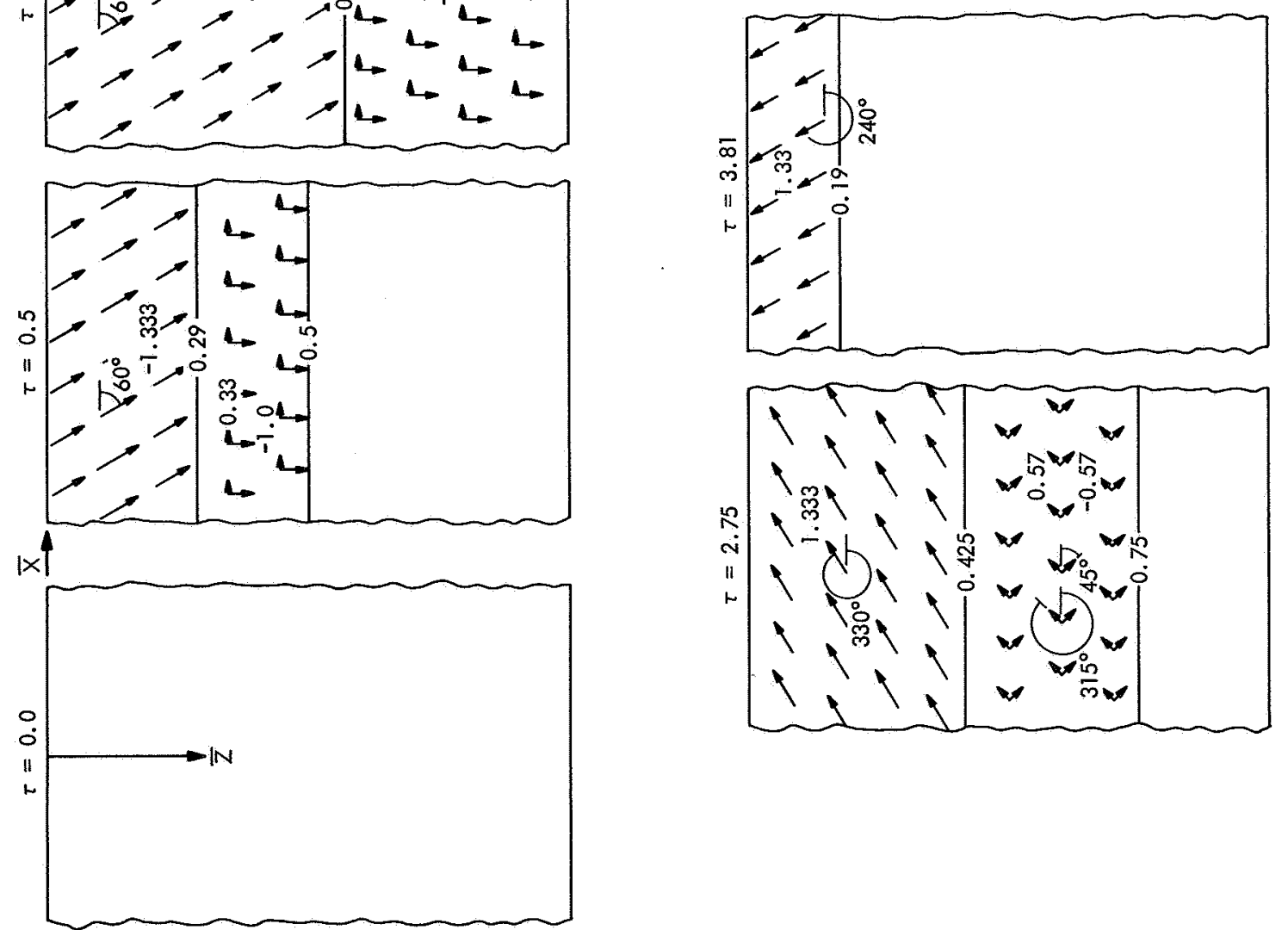
$H(\tau)= \begin{cases}0 & \tau<0 \\ 1 & \tau>0\end{cases}$

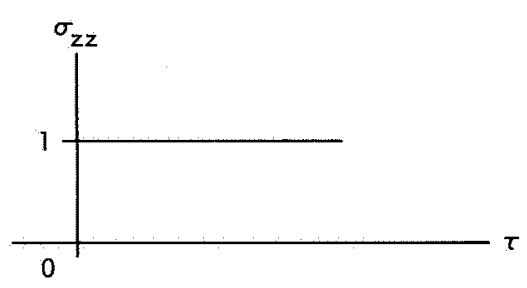

Fig. 17. Heaviside's unit step function

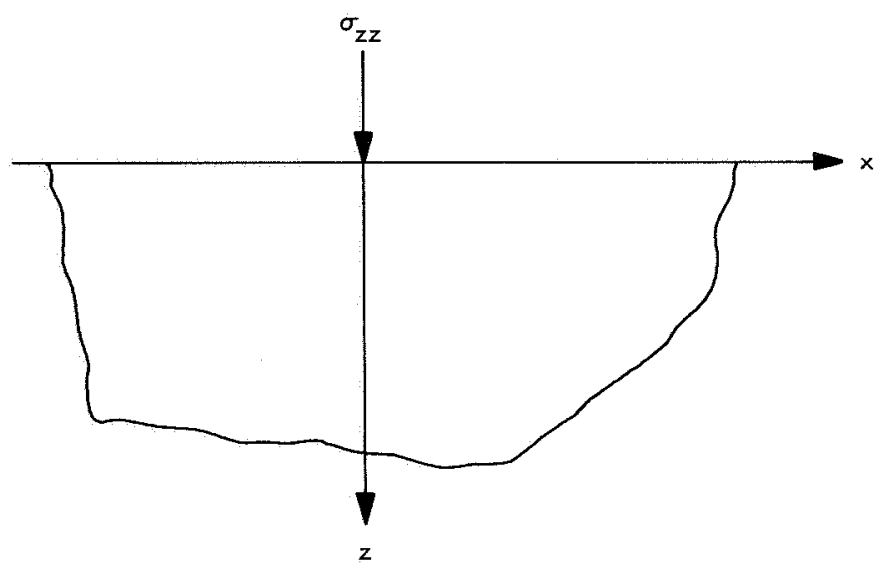

Fig. 18. Line load suddenly applied on a half-space

In view of Fig. 19, and from the Cartesian characteristic equations (68) through (72), the following relations are obtained:

For bicharacteristic curves

$\overline{A P}\left(\theta=0^{\circ}\right)$

$$
d_{\sigma_{x x}}-d U=\left(\frac{1}{3} \frac{\partial W}{\partial z}-\frac{\partial \sigma_{x z}}{\partial z}\right) d \tau
$$

$$
\text { along } \begin{aligned}
\frac{d x}{d \tau} & =1 \\
\frac{d z}{d \tau} & =0
\end{aligned}
$$

$\widehat{B P}\left(\theta=0^{\circ}\right)$

$$
\begin{aligned}
d \sigma_{x x}+d U=\left(\frac{1}{3} \frac{\partial W}{\partial z}+\frac{\partial \sigma_{x z}}{\partial z}\right) d \tau \quad & (144) \\
\text { along } \frac{d x}{d \tau} & =-1 \\
\frac{d z}{d \tau} & =0
\end{aligned}
$$

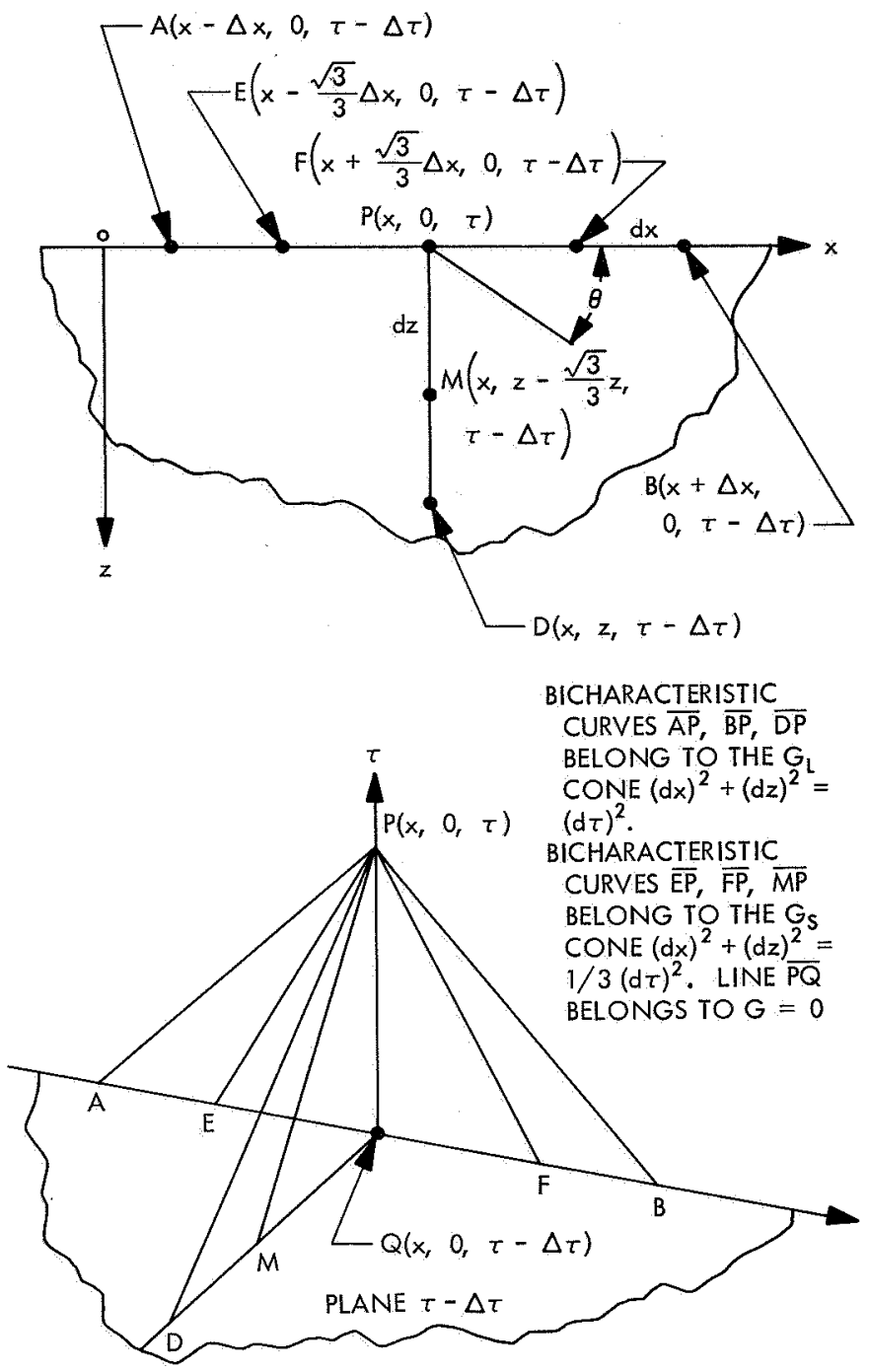

Fig. 19. Scheme to evaluate point $P(x, 0, \tau)$

$\overline{D P}\left(\theta=90^{\circ}\right)$

$$
d \sigma_{z z}+d W=\left(\frac{1}{3} \frac{\partial U}{\partial x}+\frac{\partial \sigma_{z z}}{\partial x}\right) d \tau
$$

$$
\text { along } \frac{d x}{d \tau}=0
$$

$$
\frac{d z}{d \tau}=-1
$$

$\overline{E P}\left(\theta=0^{\circ}\right)$

$$
d \sigma_{z x}-\frac{1}{\sqrt{3}} d W=\left(\frac{1}{3} \frac{\partial U}{\partial z}-\frac{1}{\sqrt{3}} \frac{\partial \sigma_{z z}}{\partial z}\right) d \tau
$$




$$
\begin{aligned}
& \overline{F P}\left(\theta=0^{\circ}\right) \\
& d \sigma_{z x}+\frac{1}{\sqrt{3}} d W=\left(\frac{1}{3} \frac{\partial U}{\partial z}+\frac{1}{\sqrt{3}} \frac{\partial \sigma_{z z}}{\partial z}\right) d \tau \quad(147) \\
& \text { along } \frac{d x}{d \tau}=-\frac{1}{\sqrt{3}} \\
& d z=0
\end{aligned}
$$

$\overline{M P}\left(\theta=90^{\circ}\right)$

$$
d \sigma_{z x}+\frac{1}{\sqrt{3}} d U=\left(\frac{1}{3} \frac{\partial W}{\partial x}+\frac{1}{\sqrt{3}} \frac{\partial \sigma_{x x}}{\partial x}\right) d \tau
$$

$$
\text { along } \begin{aligned}
\frac{d x}{d \tau} & =0 \\
\frac{d z}{d \tau} & =-\frac{1}{\sqrt{3}}
\end{aligned}
$$

For lines

$$
\begin{array}{r}
\overrightarrow{Q P}\left(\theta=0^{\circ}\right) \\
d \sigma_{z x}-3 d \sigma_{z z}=-\frac{8}{3} \frac{\partial W}{\partial z} d \tau \\
\text { along } d x=d z=0 \\
\overrightarrow{Q P}\left(\theta=90^{\circ}\right) \\
3 d \sigma_{x x}-d \sigma_{z z}=\frac{8}{3} \frac{\partial U}{\partial x} d \tau \\
\text { along } d x=d z=0
\end{array}
$$

$$
\overline{Q P}\left(\theta=0^{\circ} \text { and } \theta=90^{\circ}\right)
$$

$$
d_{\sigma_{z x}}=\left(\frac{\partial W}{\partial x}+\frac{\partial U}{\partial z}\right) \frac{d \tau}{3}
$$

$$
\text { along } d x=d z=0
$$

It is desired here to evaluate the dependent variables on the surface of the half-space $z=0$ at the leading wave front. For this purpose, the characteristic equation (143) is considered first. Because of the abrupt load, Eq. (143) is treated in a similar fashion as the preceding problem; i.e., it is written as follows ( $d \tau$ is replaced by $d x)$ :

$$
d\left[\sigma_{x x}\right]-d[U]=\left(\frac{1}{3}\left[\frac{\partial W}{\partial z}\right]-\left[\frac{\partial \sigma_{x z}}{\partial z}\right]\right) d x
$$

Across the characteristic equation (144) which holds along $d \tau=-d x$, there is no abrupt change in the dependent variables, and therefore this equation may be used as it stands. However, as $\boldsymbol{P}_{\text {front }}$ approaches $\boldsymbol{P}_{\text {rear }}$ when $d x \rightarrow 0$ (as in Fig. 13), the following relation exists (Ref. 4):

$$
\begin{gathered}
\left(\sigma_{x x_{P_{\text {rear }}}}-\sigma_{x x_{P_{\text {front }}}}\right)+\left(U_{P_{\text {rear }}}-U_{P_{\text {front }}}\right) \\
=-\int_{P_{\text {tront }}}^{P_{\text {rear }}}\left(\frac{1}{3} \frac{\partial W}{\partial z}+\frac{\partial \sigma_{x z}}{\partial z}\right) d x
\end{gathered}
$$

Since $\boldsymbol{P}_{\text {front }} \rightarrow \boldsymbol{P}_{\text {rear }}$, the right-hand side of this equation vanishes, and the following relation exists:

$$
\sigma_{x x_{P_{\text {rear }}}}=-U_{P_{\text {rear }}}
$$

or

$$
\left[\sigma_{x x}\right]=-[U]
$$

In view of Eq. (153), Eq. (152) becomes

$$
d\left[\sigma_{x x}\right]=\left(\frac{1}{6}\left[\frac{\partial W}{\partial z}\right]-\frac{1}{2}\left[\frac{\partial \sigma_{x z}}{\partial z}\right]\right) d x
$$

The first partial derivatives on the right-hand side of Eq. (154) are discontinuous derivatives of discontinuous dependent variables. Therefore, these first partial derivatives depend on the shape of the wave surface acrosswhich the dependent variables are discontinuous. Consequently, these are covariant derivatives (Ref. 15). The shape of the wave surface $\Phi$ in the $x z$-plane for an arbitrary time was found to be a circular cylinder (see Fig. 6). Since $\left[\sigma_{z z}\right]=0$ on the free surface, it follows that the covariant derivatives of the right-hand side of Eq. (154) are

$$
\left[\left(\frac{\partial W}{\partial z}\right)_{\substack{\text { covariant } \\ \text { differentiation }}}\right]=\left[\frac{\partial W}{\partial z}\right]+\frac{[U]}{r}
$$

and

$$
\left[\left(\frac{\partial \sigma_{x z}}{\partial z}\right)_{\substack{\text { covariant } \\ \text { differentiation }}}\right]=\left[\frac{\partial \sigma_{x z}}{\partial z}\right]+\frac{\left[\sigma_{x x}\right]}{r}
$$

or, by relations (26),

$$
\begin{gathered}
{\left[\left(\frac{\partial W}{\partial z}\right)_{\substack{\text { covariant } \\
\text { differentiation }}}\right]=\delta_{z} n_{z}+\frac{[U]}{r}} \\
{\left[\left(\frac{\partial \sigma_{x z}}{\partial z}\right)_{\substack{\text { covariant } \\
\text { idifferentiation }}}\right]=\delta_{x z} n_{z}+\frac{\left[\sigma_{x x}\right]}{r}}
\end{gathered}
$$


But since the analysis in this case is confined to $\theta=0^{\circ}$ (see Figs. 5 and 19), it follows that $n_{z}=0$ and $r=x$. Equation (154) is now written as follows:

$$
d\left[\sigma_{x x}\right]=\left(\frac{1}{6}[U]-\frac{1}{2}\left[\sigma_{x x}\right]\right) \frac{d x}{x}
$$

In view of Eq. (153), the above equation is written as

$$
d\left[\sigma_{x x}\right]=\left(-\frac{1}{6}\left[\sigma_{x x}\right]-\frac{1}{2}\left[\sigma_{x x}\right]\right) \frac{d x}{x}
$$

or

$$
\frac{d\left[\sigma_{x x}\right]}{\left[\sigma_{x x}\right]}=-\frac{2}{3} \frac{d x}{x}
$$

Integration of this ordinary differential equation leads to the following expression for $\sigma_{x x}$

$$
\sigma_{x x} \mid \begin{aligned}
& \text { for } z=0, x>0 \\
& \text { at leading } \\
& \text { wave front }
\end{aligned}=K x^{-2 / 3}
$$

"where the constant $K$ is taken to be equal to the initial applied load. Equation (158) becomes

$$
\sigma_{x x} \mid \begin{aligned}
& \text { on } z=0 \text { at } \\
& \text { leading } \\
& \text { wave front }
\end{aligned}=x^{-2 / 3}
$$

and, in view of Eq. (153),

$$
\left.U\right|_{\substack{\text { on } z=0, x>0 \\ \text { leading wave }}}=-x^{-2 / 3}
$$

From symmetry,

$$
\left.U\right|_{\text {on } z=0, x=0}=0
$$

Inspection of any of the characteristic equations (145, 146, and 147) shows that when $x>0$,

$$
\left.W\right|_{\substack{\text { on } z=0, x>0 \\ \text { leading wave }}}=0
$$

\section{Summary}

A method has been established by which differential equations are derived governing the propagation of 'discontinuities along the bicharacteristic curves. These differential equations were denoted in this report as the characteristic equations.

The characteristic equations were derived with reference first to Cartesian and then to cylindrical coordinate systems for a linear elastic, isotropic, and homogeneous material where plane deformation is prevalent. The applicability of these equations is confined to cases in which discontinuities may occur in the derivatives of the velocities and stresses, while these variables remain continuous.

The derived characteristic equations for two-spatial dimensions were shown to be applicable equally well to one-spatial dimensional wave problems.

Two boundary-value problems were solved using the derived characteristic equations. The first consisted of a uniform plane oblique load suddenly applied on an infinite flat plate. Velocities and stresses were obtained for the entire spatial solution domain. The second problem consisted of a line load suddenly applied on a halfspace. Velocities and stresses were obtained on the surface of the half-space at the leading wave front. In both problems, special derivations were made in order to account for possible discontinuities in velocities and stresses across the leading wave front due to the abruptly applied load. 


\section{References}

1. Fung, Y. C., Foundations of Solid Mechanics, Prentice-Hall, Inc., Englewood Cliffs, N. J., 1965.

2. Chou, P. C., and Gordon, P. F., "Radial Propagation of Axial Shear Waves in Nonhomogeneous Elastic Media," Acoust. Soc. America J., Vol. 42, No. 1, pp. 36-41, 1967.

3. Crandall, S. H., Engineering Analysis. McGraw-Hill Book Company, Inc., New York, 1956.

4. Chou, P. C., and Koenig, H. A., "A Unified Approach to Cylindrical and Spherical Elastic Waves by Method of Characteristics," J. Appl. Mech.-Am. Soc. Mech. Eng. Trans., Vol. 33, No. 1, pp. 159-168, 1966

5. Lubliner, J., "A Generalized Theory of Strain-Rate-Dependent Plastic Wave Propagation in Bars," J. Mech. Phys. Solids, Vol. 12, pp. 59-65, 1964.

6. Utku, S., On the Impact-Induced Stress Waves in Long Bars, Technical Report 32-932. Jet Propulsion Laboratory, Pasadena, Calif., 1966.

7. Hadamard, J., Leçons sur la propagation des ondes et les équations de l'hydrodynamique. Chelsea Publishing Company, New York, 1949.

8. Levi-Civita, T., Caractéristiques des systèmes différentiels et propagation des ondes. Librairie Félix Alcan, Paris, France, 1932.

9. Thomas, T. Y., "Singular Surfaces and Flow Lines in the Theory of Plasticity," J. Rational Mech. Anal., Vol. 2, pp. 339-381, 1953.

10. Garabedian, P. R., Partial Differential Equations. John Wiley and Sons, Inc., New York, 1964.

11. Thomas, T. Y., "The Decay of Waves in Elastic Solids," J. Math. Mech., Vol. 6, No. 6, pp. 759-768, 1957.

12. Lamb, H., "On the Propagation of Tremors Over the Surface of an Elastic Solid," Phil. Trans. Roy. Soc. London, Ser. A, Vol. 203, pp. 1-42, 1904.

13. Miklowitz, J., "Pulse Propagation in a Viscoelastic Solid With Geometric Dispersion," in Stress Waves in Anelastic Solids, pp. 255-276. Edited by H. Kolsky and W. Prager. Springer-Verlag, Berlin, 1964.

14. Richtmyer, R. D., and Morton, K. W., Difference Methods for Initial-Value Problems, Second Edition. Interscience Publishers, Inc., New York, 1967.

15. Sokolnikoff, I. S., Tensor Analysis, Second Edition. John Wiley and Sons, Inc., New York, 1964. 